\title{
The Shape and Density Evolution of Snow Aggregates
}

\author{
EDWin L. DunNAVAn, ZhiYuAn JiAng, Jerry Y. HARrington, AND JOHANNES VerLinde \\ Department of Meteorology and Atmospheric Science, The Pennsylvania State University, University Park, Pennsylvania \\ KyLE FitCH AND TIMOTHY J. GARRETT \\ Department of Atmospheric Sciences, University of Utah, Salt Lake City, Utah
}

(Manuscript received 18 March 2019, in final form 5 September 2019)

\begin{abstract}
Snow aggregates evolve into a variety of observed shapes and densities. Despite this diversity, models and observational studies employ fractal or Euclidean geometric measures that are assumed universal for all aggregates. This work therefore seeks to improve understanding and representation of snow aggregate geometry and its evolution by characterizing distributions of both observed and Monte Carlo-generated aggregates. Two separate datasets of best-fit ellipsoid estimates derived from Multi-Angle Snowflake Camera (MASC) observations suggest the use of a bivariate beta distribution model for capturing aggregate shapes. Product moments of this model capture shape effects to within $4 \%$ of observations. This mathematical model is used along with Monte Carlo simulated aggregates to study how combinations of monomer properties affect aggregate shape evolution. Plate aggregates of any aspect ratio produce a consistent ellipsoid shape evolution whereas thin column aggregates evolve to become more spherical. Thin column aggregates yield fractal dimensions much less than the often-assumed value of 2.0. Ellipsoid densities and fractal analogs of density (lacunarity) are much more variable depending on combinations of monomer size and shape. Simple mathematical scaling relationships can explain the persistent triaxial ellipsoid shapes that appear in both observed and modeled aggregates. Overall, both simulations and observations prove aggregates are rarely oblate. Therefore, the use of the proposed bivariate ellipsoid distribution in models will allow for similar-sized aggregates to exhibit a realistic dispersion of masses and fall speeds.
\end{abstract}

\section{Introduction}

Snow aggregates represent a dominant proportion of observed ice precipitation on Earth yet the processes that dictate formation and structure are still poorly understood (Connolly et al. 2012). Each aggregate represents a connected ensemble of individual ice particles, each called a "monomer," that have collided and stuck to one another while falling. The resulting snow aggregate geometry, representing the totality of all constituent monomers and their particular configuration, is important for accurately describing and predicting snow precipitation rates (Heymsfield and Westbrook 2010; Westbrook and Sephton 2017), collection rates (Mitchell 1988), and radiation properties (Petty and Huang 2010). Euclidean objects are commonly used as volumetric shells when calculating these various physical properties. In general, Euclidean geometry describes how

\footnotetext{
Corresponding author: Edwin L. Dunnavan, eld172@psu.edu
}

many independent dimensions are required to specify any point on a particular Euclidean object: Cubes require three dimensions, planes require two dimensions, and lines require only one dimension. Therefore, Euclidean objects exhibit logarithmic scaling based on each integer dimension. Making each object twice as large requires $2^{3}=8$ identical copies for a cube, $2^{2}=4$ identical copies for a plane, and $2^{1}=2$ identical copies for a line. This simplicity provided by Euclidean geometry allows for simple calculations of 3D quantities, such as mass, as well as $2 \mathrm{D}$ quantities, such as projected area which is often used in fall speed calculations.

Current microphysics models often assume spheres or oblate spheroids as Euclidean proxies (e.g., Thompson et al. 2008; Morrison and Milbrandt 2015; Jensen et al. 2017) although other models incorporate more complex geometries (e.g., Hashino and Tripoli 2011a,b). An assumed density couples volume with mass, thereby completing an aggregate's geometry. In general, models use power-law relations that scale density with length. 
Therefore, Euclidean geometry permits the algebraic consolidation of density and volume when formulating mass:

$$
m_{i}=\rho_{i} V_{i}=\left(\alpha_{\rho} D^{\beta_{\rho}}\right)\left(\alpha_{V} D^{\beta_{V}}\right)=\alpha_{m} D^{\beta_{m}}
$$

where $\alpha_{m}=\alpha_{\rho} \alpha_{V}, \beta_{m}=\beta_{\rho}+\beta_{V}$, and $D$ is a length scale; $\alpha_{m}$ and $\beta_{m}$ are coefficients that can be derived from fits to in situ data (e.g., Locatelli and Hobbs 1974; Mitchell 1996). The consolidation of density and volume therefore yields a well-defined physical representation for the geometry of each aggregate: the Euclidean volume $V_{i}$ represents the spatial extent of the aggregate's mass whereas the density $\rho_{i}$ represents how much of $V_{i}$ is filled with ice. Equation (1) is well suited for microphysics models since integration of these power-law functional relations over gamma distributed particle lengths yields gamma functions. However, bulk microphysics models will often employ mass-dimensional relationships where each constant represents a combination of both shape and density (e.g., Brown and Francis 1995). This convolution obfuscates the relative contribution of each component to $\alpha_{m}$ and $\beta_{m}$. Current theories that mathematically link an aggregate's morphology to its physical properties, such as that developed by Böhm (1989), are predicated on appropriately segregating shape and density. However, the Euclidean concepts of "shape" and "density" themselves suggest some sort of structural consistency; such consistency is almost nonexistent in observed aggregates. Various monomer configurations can produce wildly different physical properties. Confined configurations can reduce drag through the reduction of surface area relative to fall direction. Conversely, "open" configurations, such as linear chains, can increase this same surface area, thus producing an opposite effect on fall speed.

Despite aggregate diversity, both observations and numerical studies support claims of geometric universality. For instance, the Monte Carlo aggregate model of Westbrook et al. (2004a) produces mean projected aspect ratios that asymptote toward a value of about 0.6 . This value is remarkably consistent with the $0.6-0.8$ observational estimates from Korolev and Isaac (2003), Brandes et al. (2007), and Garrett et al. (2015) as well as radar scattering calculations from Matrosov et al. (2005) and Hogan et al. (2012). While models have used these aspect ratio values to characterize aggregates, the relationship between these values and their assumed underlying shape has remained ambiguous. Projection uncertainties compound this ambiguity when orientations sufficiently distort 2D observations away from the true 3D aggregate structures. The correct distribution of aggregate orientations, while still largely uncertain, is thought to depend upon both environment and aggregate morphology (see Pruppacher and Klett 1997).

This orientation uncertainty led Jiang et al. (2017) to test the implications of projecting spheroids in a way that is analogous to observations. Their tests showed that spheroids appear much more spherical than their true 3D aspect ratios. Therefore, if aggregates are well represented by oblate spheroids then their mean aspect ratios are actually much lower than what their oftenassumed values of 0.6 or 0.8 would suggest. In fact, analytical solutions of projected aspect ratio distributions from gamma distribution axis lengths are not only sensitive to orientation, but also to the size distribution shape factor $\nu$ (Dunnavan and Jiang 2019). The results of these studies suggest that the projection process itself ruins any attempt at inferring $3 \mathrm{D}$ geometry from singleview aggregate imaging.

Fortunately, recent developments in imaging technology permit much better $3 \mathrm{D}$ aggregate reconstructions. For instance, the Multi-Angle Snowflake Camera (MASC) (Garrett et al. 2015) can simultaneously image aggregates from multiple viewing angles; the use of three or more viewing directions greatly reduces uncertainties associated with 3D retrievals (Kleinkort et al. 2017). A recent study by Jiang et al. (2019) used the MASC to estimate best-fit aggregate ellipsoids. They found that prolate spheroids actually better characterized aggregates than oblate spheroids. Furthermore, aggregates were often preferentially canted based on turbulent intensity rather than oriented horizontally as is often assumed. Overall, the inconsistency in prior assumptions regarding aggregate shape and orientation combats the assumed universal measures. Projection uncertainties therefore present a fundamental challenge when estimating 3D aggregate geometry: a single set of geometric relationships derived from observations cannot necessarily describe multiple aggregate properties. Improving aggregate representation in models therefore requires multiple sets of geometric measures in calculations or an entirely new paradigm altogether.

One such paradigm has been to describe aggregate geometry using a fractal approach (e.g., Westbrook et al. 2004a,b; Maruyama and Fujiyoshi 2005; Ishimoto 2008; Schmitt and Heymsfield 2010). This fractal approach takes Eq. (1) in its convolved form and assumes that each aggregate mass scales according to the constituents of each monomer. The fractal description therefore circumvents the issues of defining or estimating shape and density individually. Boxcounting methods can be used to estimate a fractal dimension $D_{f}$. These boxcounting methods overlay multiple $3 \mathrm{D}$ grids of varying resolutions onto an object. Monomer elements are counted within each grid box such that the number of 
boxes with at least one element increases roughly as a power-law with grid length. The slope of this power law represents $D_{f}$, which serves as $\beta_{m}$ in Eq. (1). Using this boxcounting approach, Schmitt and Heymsfield (2010) found that a scaling factor, $S \approx 1.30$, could sufficiently relate $3 \mathrm{D}$ fractal dimensions of simulated aggregates to the 2D fractal dimensions calculated from their various projections. However, the use of a constant $S$ for estimating $D_{f}$ from in situ images still has some significant drawbacks. Even small deviations in $S(\Delta S=0.02)$ can lead to substantial changes in bulk quantities $(10 \%$ change in ice water content; Schmitt and Heymsfield 2010). While the lack of eccentric particles at lower temperatures acts to restrict the range of $S$ values (see Table 1 in Schmitt and Heymsfield 2010), the presence of dendrites and needles at higher temperatures (Bailey and Hallett 2009) could lead to larger deviations in $S$ for snow aggregates. Furthermore, the methodology of Schmitt and Heymsfield (2010) still relies on one-to-one mass-dimensional relationships which do not by themselves capture the correct dispersion of particle properties like fall speeds (Passarelli 1978a,b; Sasyo and Matsuo 1985). Schmitt and Heymsfield (2010) also found that the observed 3D mean fractal dimension itself varied from about 2.0 to 2.3 with an apparent dependence on temperature. Other authors (e.g., Heymsfield et al. 2004; Westbrook et al. 2004a,b) have reported similar values for $D_{f}$ that fall well within the range of Schmitt and Heymsfield (2010). Despite the observed $D_{f}$ variability as found by Schmitt and Heymsfield (2010) and others, some studies have maintained $D_{f} \approx 2.0$ as a universal geometric feature of aggregates (e.g., Westbrook et al. 2004a,b; Stein et al. 2015) or have treated aggregates as such when developing mass dimensional relationships from observational datasets (e.g., Brown and Francis 1995; Mitchell 1996).

Fractal dimensions however are not the only type of fractal measure. For instance, $D_{f}$ can be generalized as a multifractal dimension $D_{q}$. To do this, a partition function rather than a binary unit is used to describe the boxcounting spatial information at various grid scales. Moments of this partition function across various grid sizes can help infer whether an object is nonfractal, monofractal, or multifractal. Positive moments of the partition function amplify denser regions of the aggregate whereas negative moments amplify sparser regions (Chhabra and Jensen 1989). The amplification effect of various moments therefore can provide more information about the spatial distribution of aggregate mass.

Another important fractal quantity is lacunarity. This quantity shares the same etymology as "lacunae," which is occasionally used to describe the hollowing of ice crystal columns or the gaps in between pristine dendrite branches (see Nelson 2001). Similarly, lacunarity can be loosely thought of as the "gappiness" of a particular fractal; that is, lacunarity represents the size distribution of holes throughout a particular fractal. In this sense, lacunarity is a measure of porosity or density where higher values of lacunarity represent more gaps overall. Furthermore, fractals with low values of lacunarity exhibit more rotational or translational invariance, whereas fractals with high values of lacunarity are more sensitive to orientation. One can imagine lacunarity as a densitylike measure that appropriately scales aggregate mass with size; as aggregates grow, so should the number of gaps in between monomers. Lacunarity can also be used to evaluate the invariant or variant nature of how $2 \mathrm{D}$ images or projections of aggregates correspond to their 3D geometries. Therefore, lacunarity could be helpful for supplementing the in situ methodology of Schmitt and Heymsfield (2010). In general, aggregate features (e.g., maximum dimension, area ratio, and fractal dimension) are assumed to be more or less spatially invariant. As far as we are aware, however, this assumption has not been tested for various types of aggregates and it is not clear whether certain types of aggregates yield higher values of lacunarity than others. We test this assumption herein.

Lacunarity can also provide more depth for fractal analyses. For instance, Mandelbrot (1994) showed that Cantor dust sets of the exact same fractal dimension can still have features that differ "violently" from one another. Therefore, fractal dimensions alone might not sufficiently capture multiple physical properties for each aggregate if mass varies significantly for a particular size. The effect of density variations among various aggregates can be inferred from Fig. 8 in Erfani and Mitchell (2016) which shows about an order of magnitude spread in estimated mass for any particular aggregate size. This large spread in estimated mass across an entire size distribution could compound errors in other estimates, such as fall speed, that depend on both the total amount of mass and its spatial distribution. Additionally, Mandelbrot (1992) and Blumenfeld and Mandelbrot (1997) show that certain lacunarity quantities can be used to estimate $\alpha_{m}$ in Eq. (1). These techniques could be similarly applied to snow aggregates as well to better constrain Eq. (1) prefactors.

Despite large uncertainties associated with estimating aggregate geometry from projections and assigning these measures to a single length scale, both Euclidean and fractal descriptions of aggregates seem to yield consistent or universal values: Observations using both in situ observations (e.g., Korolev and Isaac 2003) and radar calculations (e.g., Hogan et al. 2012) suggest a universal aspect ratio of 0.6 , whereas theoretical studies (e.g., Westbrook et al. 2004a,b) and in situ 


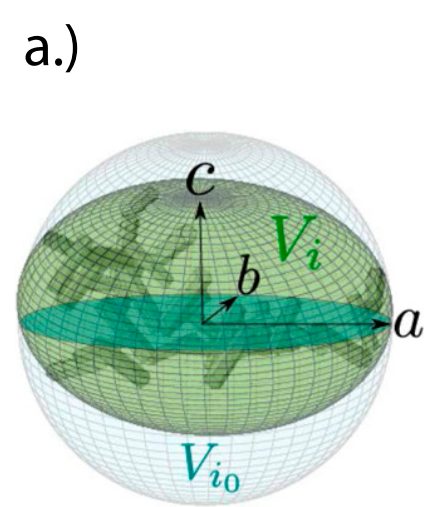

$a \geq b \geq c$

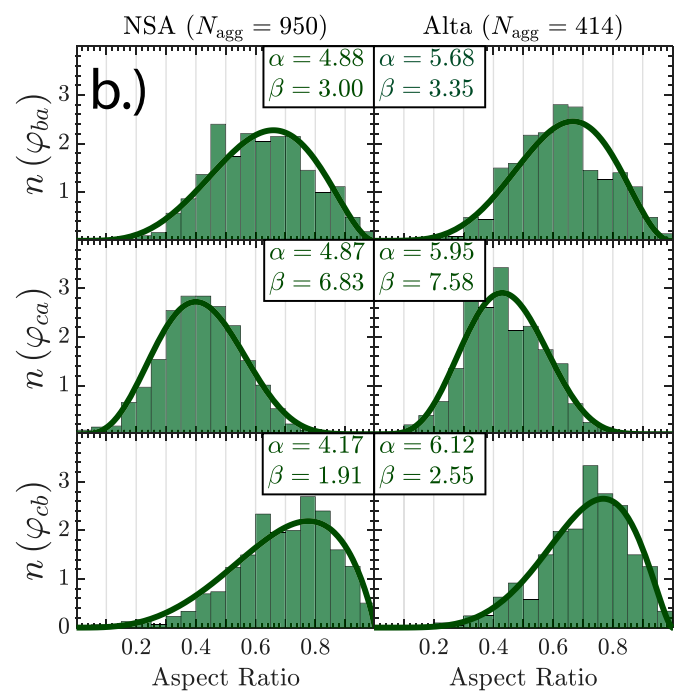

FIG. 1. (a) Modeled aggregate image from Fig. 12 of Westbrook et al. (2008) conceptualized as a reduced density ellipsoid of volume $V_{i} . V_{i_{0}}$ represents a reduced density sphere of the same maximum dimension, $2 a$. (b) Ellipsoidal aggregate aspect ratio probability distributions derived from the MASC imager following Jiang et al. (2019) for $\varphi_{b a}=b / a, \varphi_{c a}=$ $c / a, \varphi_{c b}=c / b$, where $a \geq b \geq c$. Histograms are shown for (left) all 950 ellipsoids derived from the NSA MASC aggregate database and (right) all 414 ellipsoids derived from the Alta MASC aggregate database. Beta distribution fits are shown as green lines, where the fitted beta distribution parameters $\alpha$ and $\beta$ are shown for each aspect ratio, rounded to two decimal places.

(e.g., Schmitt and Heymsfield 2010) and radar (e.g., Stein et al. 2015) observations suggest a universal fractal dimension of $D_{f} \approx 2.0$. The question we ask is whether these results are physical or coincidental. Does each individual snow aggregate evolve into a truly universal shape and density, or, in the language of Mandelbrot (1994), do certain "chimeras" of the same fractal dimension differ "violently" from one another?

Previous aggregate Monte Carlo modeling studies such as Westbrook et al. (2004a,b); Maruyama and Fujiyoshi (2005) not only simulated the evolution of aggregate sizes and shapes, but also the evolution of aggregate fall speeds and size distributions. This study intentionally severs this link. Instead, we focus on how Euclidean descriptions of shape and density and estimates of fractal quantities evolve for different types of monomer geometries and their combinations. We can then use the resulting distributions of aggregate geometry to infer the statistical properties of all aggregates. For simplicity, we assume each monomer is well described by spheroidal geometry (i.e., $\varphi_{\text {mon }}=$ $c / a$ and $\rho_{i}=$ const). With these assumptions, we attempt to link a priori shape and density information of primary habits (i.e., planar and columnar particles) to the resulting Euclidean and fractal evolution of their aggregates.

\section{Observations}

To provide a basis for our Monte Carlo simulations (sections 3 and 4), we first present recent aggregate observations of estimated ellipsoid shapes derived from the MASC imager. The gradient descent method used herein to estimate these observed ellipsoid aspect ratios is described in detail by Jiang et al. (2019). Figure 1a shows a schematic of a best-fit ellipsoid superimposed onto an aggregate. For consistency, we assume each ellipsoid dimension is arranged as: $a \geq b \geq c$. Notice that two aspect ratio measures are required to specify the ellipsoid shape although it is not clear which ratios are the most convenient. For this reason, we define the following aspect ratios:

$$
\begin{aligned}
\varphi_{b a} & \equiv \frac{b}{a}, \\
\varphi_{c a} & \equiv \frac{c}{a}, \\
\varphi_{c b} & \equiv \frac{c}{b} .
\end{aligned}
$$

The ellipsoid volume can therefore be neatly expressed as

$$
V_{i}=\frac{4}{3} \pi a b c=\frac{4}{3} \pi a^{3} \varphi_{b a} \varphi_{c a}=V_{i_{0}} \varphi_{b a} \varphi_{c a}
$$


or

$$
V_{i}=\frac{4}{3} \pi a b c=\frac{4}{3} \pi a^{3} \varphi_{b a}^{2} \varphi_{c b}=V_{i_{0}} \varphi_{b a}^{2} \varphi_{c b}
$$

Notice that the ellipsoid volume $\left(V_{i}\right.$, green shell in Fig. 1a) is related to a spherical volume of radius $a\left(V_{i_{0}}\right.$, blue shell in Fig. 1a) by aspect ratio scaling factors.

Figure 1b shows a comparison of the estimated ellipsoid aspect ratio distributions for snow aggregates observed at two separate locations: The North Slope of Alaska (NSA) and Alta, Utah (Alta). Each row represents the probability distribution $n(\varphi)$ for the three different aspect ratio definitions in Eq. (2). The distributions of these aspect ratios seem to be rather well behaved; each distribution is unimodal with fewer counts observed at the extremes: $\varphi \rightarrow 0$ and $\varphi=1.0$. The form of the distributions is consistent with that of the beta distribution which is described by the following probability density function

$$
n(\varphi)=\frac{1}{B(\alpha, \beta)} \varphi^{\alpha-1}(1-\varphi)^{\beta-1} \Rightarrow \varphi \sim \operatorname{Beta}(\alpha, \beta),
$$

where $B(\alpha, \beta)$ is the beta function. Beta distribution fits over these distributions visually capture the distribution shapes for each dataset. To test the similarity between each dataset, we performed a two-sample KolmogorovSmirnov test on each dataset. For each aspect ratio, $\varphi_{b a}$, $\varphi_{c a}$, and $\varphi_{c b}$, the null hypothesis is not rejected at the $5 \%$ significance level $(p=0.1144, p=0.0821$, and $p=0.0527$, respectively). Welch's $t$ test gives confidence intervals for the difference in population means $\left(\bar{\varphi}_{\text {NSA }}-\bar{\varphi}_{\text {Alta }}\right)$ of $(-0.0289,0.0072),(-0.0364,-0.0058)$, and $(-0.0352,0.0025)$, respectively. Therefore, while the distributions themselves are not necessarily identical, the distribution means of each aspect ratio are consistent.

The consistency of the beta-like marginal distributions of $\varphi_{b a}, \varphi_{c a}$, and $\varphi_{c b}$ with that of the observations (Fig. 1) suggests the use of a bivariate beta distribution to describe all ellipsoids. Here, we use the first bivariate beta distribution given in Nadarajah and Kotz [2005, their Eq. (4)]. This bivariate distribution is specified by the relationships

$$
\begin{aligned}
& \varphi_{c a}=\varphi_{b a} \varphi_{c b}, \\
& \varphi_{b a}=\varphi_{b a},
\end{aligned}
$$

where Lemma 1 from Nadarajah and Kotz (2005) is used to specify the following beta distributions: $\varphi_{b a} \sim \operatorname{Beta}\left(\alpha_{b a}\right.$, $\left.\beta_{b a}\right), \varphi_{c a} \sim \operatorname{Beta}\left(\alpha_{b a}, \beta_{b a}+\beta_{c b}\right)$, and $\varphi_{c b} \sim \operatorname{Beta}\left(\alpha_{b a}+\right.$ $\left.\beta_{b a}, \beta_{c b}\right)$. These definitions specify the following bivariate distribution for $\varphi_{b a}$ and $\varphi_{c a}$ :

$$
\begin{aligned}
n\left(\varphi_{b a}, \varphi_{c a}\right)= & \frac{1}{B\left(\alpha_{b a}, \beta_{b a}\right) B\left(\alpha_{b a}+\beta_{b a}, \beta_{c b}\right)} \varphi_{c a}^{\alpha_{b a}+\beta_{b a}-1} \\
& \times\left(\varphi_{b a}-\varphi_{c a}\right)^{\beta_{c b}-1} \varphi_{b a}^{-\beta_{b a}-\beta_{c b}}\left(1-\varphi_{b a}\right)^{\beta_{b a}-1}
\end{aligned}
$$

This particular bivariate distribution has triangular support: $0<\varphi_{c a} \leq \varphi_{b a}<1.0$. The nice aspect of Eq. (7) is that product moments are represented in terms of complete beta functions

$$
\begin{aligned}
\left\langle\varphi_{b a}^{m} \varphi_{c a}^{n}\right\rangle & \equiv E_{m n} \\
& =\frac{B\left(m+n+\alpha_{b a}, \beta_{b a}\right) B\left(n+\alpha_{b a}+\beta_{b a}, \beta_{c b}\right)}{B\left(\alpha_{b a}, \beta_{b a}\right) B\left(\alpha_{b a}+\beta_{b a}, \beta_{c b}\right)},
\end{aligned}
$$

where $m$ and $n$ represent distribution moments. These beta functions can be represented in terms of gamma functions

$$
B(\alpha, \beta)=\frac{\Gamma(\alpha) \Gamma(\beta)}{\Gamma(\alpha+\beta)}
$$

Furthermore, integer product moments of Eq. (8) can be expressed algebraically using properties of the gamma function. This means that the method of moments technique can be used to estimate the three parameters: $\alpha_{b a}, \beta_{b a}$, and $\beta_{c b}$. Doing this for $E_{10} \equiv \bar{\varphi}_{b a}, E_{01} \equiv \bar{\varphi}_{c a}$ and $E_{11} \equiv\left\langle\varphi_{b a} \varphi_{c a}\right\rangle$ yields simple, algebraic expressions for each parameter

$$
\begin{aligned}
& \alpha_{b a}=\frac{E_{10} E_{01}-E_{10} E_{11}}{E_{11}-E_{01} E_{10}}=\bar{\varphi}_{b a}\left[\frac{\bar{\varphi}_{c a}\left(1+\bar{\varphi}_{b a}\right)}{\operatorname{cov}\left(\varphi_{b a}, \varphi_{c a}\right)}-1\right], \\
& \beta_{b a}=\alpha_{b a}\left(\frac{1}{E_{10}}-1\right)=\alpha_{b a}\left(\frac{1}{\bar{\varphi}_{b a}}-1\right) \\
& \beta_{c b}=\alpha_{b a}\left(\frac{1}{E_{01}}-1\right)-\beta_{b a}=\alpha_{b a}\left(\frac{1}{\bar{\varphi}_{c a}}-1\right)-\beta_{b a},
\end{aligned}
$$

where $\operatorname{cov}(X, Y)$ represents covariance.

Figure 2 shows a comparison of the bivariate distribution from the NSA MASC and our bivariate beta distribution model fitting using Eqs. (7) and (10a)-(10c). Despite the simplicity of this method of moments approach, the bivariate model seems to do a remarkable job capturing the entire ellipsoid parameter space. To further illustrate the effectiveness of our mathematical model, Fig. 3 shows how product moments of our model compare to those estimated from the MASC derived ellipsoid database for NSA and the resulting relative error. Product moments are shown from 0 to 2 since reflectivity is often considered to be proportional to $m=n=2$ and bulk fall speed quantities represent fractional moments 


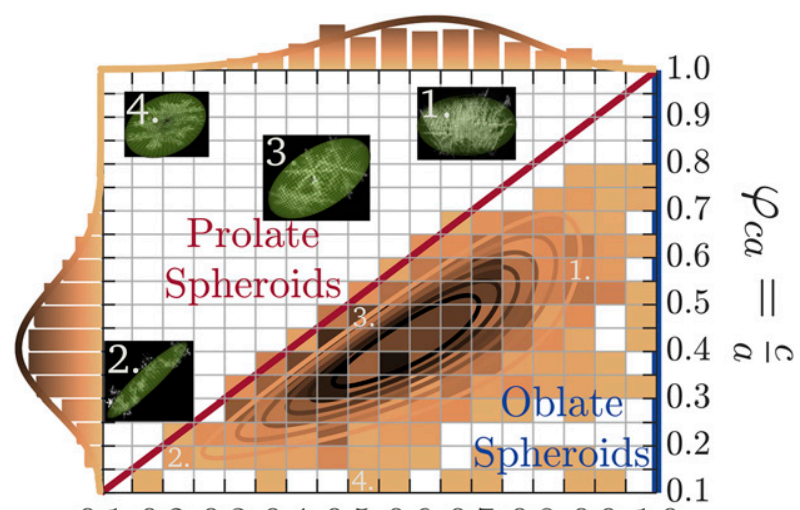

$\begin{array}{lllllllllll}0.1 & 0.2 & 0.3 & 0.4 & 0.5 & 0.6 & 0.7 & 0.8 & 0.9 & 1.0\end{array}$

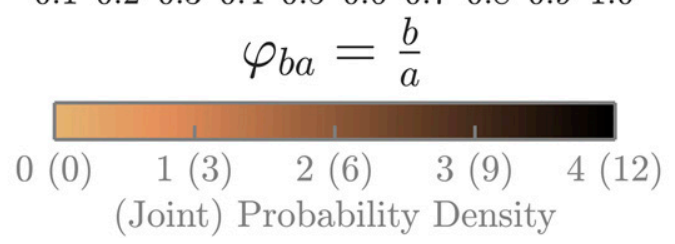

FIG. 2. Joint probability density function of the NSA MASC derived ellipsoid aspect ratios $\varphi_{b a}$ and $\varphi_{c a}$ and associated marginal distributions (shaded histograms). Solid lines represent the (joint) probability distribution dictated by Eqs. (7) and (10). Four examples of MASC aggregates and their best-fit ellipsoids are shown along with their locations on the joint histogram.

anywhere from $m=n=0$ to $m=n=2$ depending on each aggregates' Reynolds number. Aggregate orientations make $m \neq n$ through changing projected areas. Overall, product moments of our model are within only $4 \%$ of that estimated from the MASC ellipsoids. These tests indicate that Eqs. (7) and (10) provide a sufficient mathematical basis to describe distributions of aggregates as represented by ellipsoids. In the rest of this paper we use these equations in conjunction with Monte Carlo simulations to examine the evolution of aggregate shapes. While the MASC datasets represent ground-based observations, the entire layer below cloud top was frequently at ice saturation and thus produced aggregates in many stages of evolution (see example images in Fig. 2). Riming was evident for aggregates in both datasets, but we do not explicitly including riming for our Monte Carlo simulations. Instead, we expect that riming will either fill in gaps in the aggregate, thereby decreasing lacunarity and increasing density, or make monomer shapes more spherical. Therefore, riming effects on ellipsoid shapes are assumed implicit in the characterization of monomer aspect ratios.

\section{Methods}

\section{a. Monte Carlo aggregation method}

Initial monomers are specified as spheroids (either oblate, prolate, or sphere) with aspect ratios of $\varphi_{\text {mon }}=c / a$.
For these spheroidal monomers, we follow the convention of Chen and Lamb (1994) where $\varphi_{\text {mon }}<1.0$ (i.e., $b=a$ in Fig. 1a) are oblate and $\varphi_{\text {mon }}>1.0$ (i.e., $b=c$ in Fig. 1a) are prolate. Each monomer is itself made up of individual sphere elements (which we call "dipoles") with location $(x, y, z)$ and diameters of unity (arbitrary units). These dipoles also act as lattice sites upon each monomer where additional monomer dipole elements can attach. Each aggregation event therefore constitutes selecting an orientation for each collecting species, projecting each of the dipole elements onto the $x-y$ plane, and randomly selecting lattice sites on each species based on each projection. Dipoles that are completely surrounded by other dipoles are not chosen as attachment sites. The maximum dimension of the aggregate ensemble is calculated after each aggregation event and this length is then rotated to fall along the $x$ axis using an Euler angle rotation matrix (see Jiang et al. 2017). This maximum dimension is defined to be twice the $a$-axis length of the best-fit ellipsoid. The $b$ and $c$ axes are calculated by fitting ellipses around the 2D projections in the $x-y$ and $y-z$ planes. The ellipse fits are determined by matching the second central moment of the fitted ellipse to the projected areas from each dipole. This means that aggregate dipoles can have locations outside the ellipsoid shell. Unless otherwise noted, particles are assumed to be randomly oriented during collection.

Previous Monte Carlo studies (e.g., Westbrook et al. 2004a,b; Maruyama and Fujiyoshi 2005; Schmitt and Heymsfield 2010) provide a more physical and realistic representation for aggregation by directly calculating a hydrodynamic collection kernel associated with individual particles sticking together. To do this, these studies either use the methodology of Gillespie (1975), which assumes a time scale associated with each collection event (e.g., Maruyama and Fujiyoshi 2005) or explicitly calculate particle trajectories (e.g., Westbrook et al. 2004a,b; Schmitt and Heymsfield 2010). These simulations directly predict quantities associated with microphysical variables such as aggregation efficiency $E_{\mathrm{agg}}$, which describes the probability that two colliding particles will stick, differential fall speed $\Delta v_{t}$ and the total time-averaged projected area provided by both collecting particles, $\left(\sqrt{A_{x}}+\sqrt{A_{y}}\right)^{2}$ (Connolly et al. 2012). The aggregation kernel, described by the direct multiplication of these terms, represents a statistical average collection rate for the totality of the aggregation physics. Our Monte Carlo simulations, by contrast, can include different types of aggregation events implicitly and is thus quite general for representing various forms of aggregation (i.e., aggregation in clouds versus snow aggregation toward the ground). The goal of our simplified approach is not to precisely determine the resulting microphysical evolution of each individual aggregate but to capture an appropriate evolution of their 

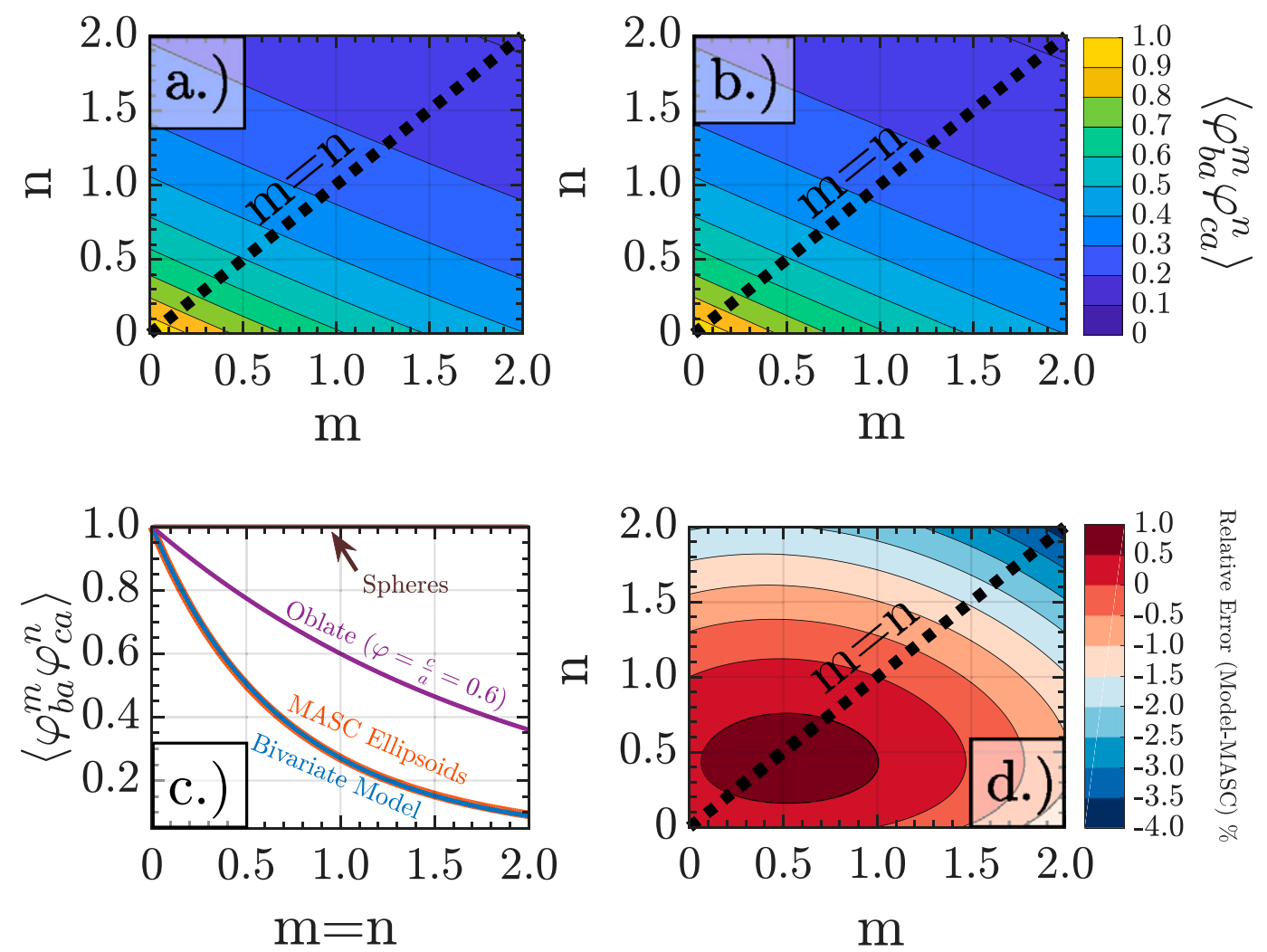

FIG. 3. Comparison of distribution moments for MASC-derived ellipsoids from (a) NSA and (b) the bivariate beta distribution model. (c) Product moments (or spheroid $\varphi_{c a}$ moments) when $m=n$ for spheres, oblate spheroids ( $\varphi=c / a=0.6$ ), MASC-derived ellipsoids, and the bivariate aspect ratio ellipsoid model. (d) Relative error of the ellipsoid aspect ratio product moment between MASC derived aggregates and the bivariate model.

shapes and densities based on various monomer types. Further details such as the particle fall speeds and size distributions are likely to affect the asymptotic characteristics when the number of monomers increases (see Westbrook et al. 2004a,b) and these details could influence the resulting aggregate geometries.

\section{b. Calculation of fractal properties}

Generalized fractal dimensions for simulated aggregates are computed using a boxcounting method similar to Karperian (2013) but generalized even further for multiple model runs. Each generated aggregate has monomer dipole locations that are stored in terms of their Cartesian coordinates and binned for each boxcounting grid size $l$. For each aggregate, multiple successive grids are set up by using the ellipsoid maximum dimension, $2 a$, to determine the initial grid length. Figure 4 shows an example of the boxcounting procedure using different numbers of grid boxes for a given aggregate. Each grid is specified by a grid length $l$, which divides the aggregate into $N_{\text {grid }}=2^{n}$ components and creates a total of $2^{n}$ total boxes for each $x, y$, and $z$ direction. This gives a total of $2^{3 n}$ boxes for each aggregate where the length scale $l$ is given by $l=2^{-n}$. The common definition for the generalized multifractal dimension is given by

$$
D_{q}=\frac{1}{q-1} \lim _{l \rightarrow 0} \frac{\log [Z(q, l)]}{\log l} .
$$

$Z(q, l)=\sum_{i=1}^{N_{\text {agg }}} P_{i, l}^{q}$ is a partition function where dipole probabilities $P_{i, l}$ are given by $P_{i, l}=\left.\left(N_{i} / N_{\text {agg }}\right)\right|_{l}$, and $q$ is a particular moment; $N_{i}$ is the total number of dipoles with locations $\left(x_{i}, y_{i}, z_{i}\right)$ located in a bin with grid resolution $l$ that makes up the total number of dipoles for a particular aggregate $N_{\text {agg. }}$. Equation (11) however is difficult to use in practice since the limit $l \rightarrow 0$ cannot be calculated numerically. Instead, the more common approach is to utilize L'Hospital's rule to approximate this limit at a physically relevant and computationally reasonable grid scale $l_{0}$ :

$$
\left.D_{q} \approx \frac{1}{q-1} \frac{\partial \log [Z(q, l)]}{\partial \log l}\right|_{l=l_{0}}
$$

To evaluate Eq. (12), $D_{q}$ is plotted for each $q$ value as a function of each grid size $l$ around $l=l_{0}$. From this plot, $D_{q}$ can be estimated in terms of the slope of the linear 

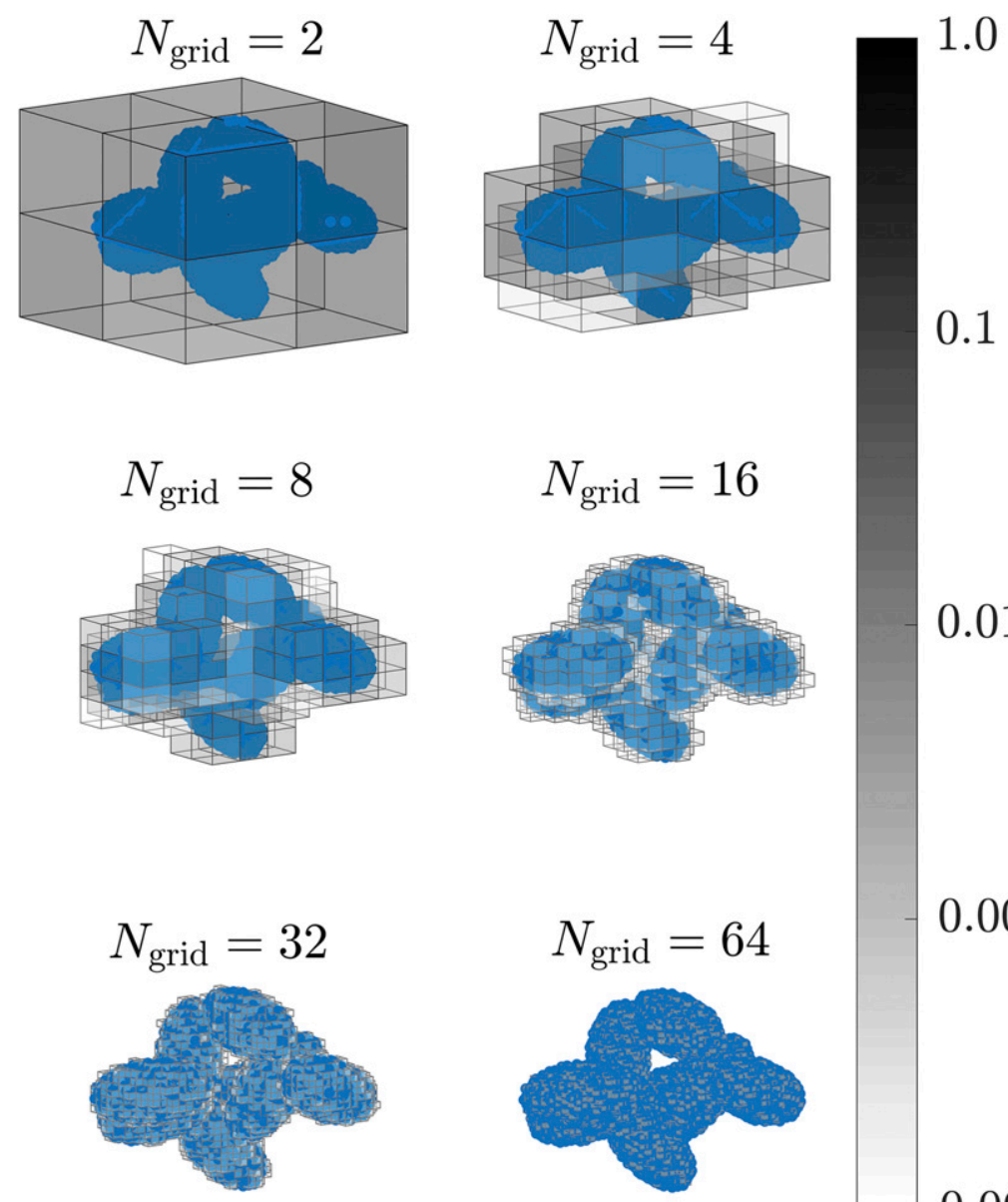

0.001

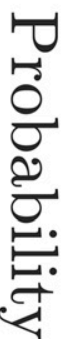

FIG. 4. Example of generalized boxcounting procedure. For visual simplicity, box outlines are removed when the probability is zero.

regression in $\log -\log$ space. For $q=0$, the generalized fractal dimension is equal to the (mono) fractal dimension or boxcounting dimension defined by

$$
D_{f} \equiv D_{0}=\left.\frac{\partial \log N}{\partial \log l}\right|_{l=l_{0}} \Rightarrow N \propto l^{-D_{f}},
$$

where $N$ represents the total number of boxes that has at least one dipole. Throughout this paper, we refer to $D_{0}$ as $D_{f}$ for consistency with previous works (e.g., Westbrook et al. 2004b; Schmitt and Heymsfield 2010). For $q=1$, Eq. (11) corresponds to the information (entropy) dimension. ${ }^{1}$ For $q=2$, Eq. (11) corresponds to the "correlation dimension" (Karperian 2013).

\footnotetext{
${ }^{1}$ Setting $q=1$ yields a singularity using Eq. (11). However, this singularity can be avoided by using L'Hospital's rule. The following equation gives the appropriate information dimension: $D_{1}=\lim _{l \rightarrow 0}\left(\sum_{i=1}^{N_{\text {dip }}} P_{i, l} \log P_{i, l} / \log l\right)$ (see van Opheusden et al. 1996).
}

While boxcounting methods for estimating generalized fractal dimensions are calculated in a standard way, calculations of lacunarity have historically been performed using a variety of methods (Smith et al. 1996). In fact, Mandelbrot (1994) stated, "I have little hope of finding a unique measure of lacunarity." For this work, we characterize lacunarity as the efficiency, or the square of the coefficient of variation $(\mathrm{CV})$ of the multifractal partition function:

$$
\lambda_{l} \equiv \mathrm{Eff}_{l}=\left(\mathrm{CV}_{l}\right)^{2}=\left(\frac{\sigma_{l}}{\mu_{l}}\right)^{2}=\frac{Z(2, l)}{[Z(1, l)]^{2}}-1
$$

where $\sigma_{l}$ represents the standard deviation of dipole probabilities for each model run, and $\mu_{l}$ represents the mean of dipole probabilities for each model run. Notice that the form of Eq. (14) provides a nice conceptual relationship with the multifractal moments $q=1$ and $q=2$. In general, lacunarity is orientation dependent which means that different aggregate orientations will yield different values of $\lambda_{l}$. As a result, it is common to 
sample and average $\lambda_{l}$ over multiple grid orientations. Therefore, an average lacunarity can be calculated as

$$
\Lambda=\frac{1}{G L} \sum_{g=1}^{G} \sum_{l=1}^{L} \lambda_{l, g}
$$

where $l$ represents each grid and $g$ represents each orientation. We found in our initial tests that averaging over multiple particle orientations did little to change fractal quantities when we had many model runs. Rather, the spread in fractal quantities was dominated by the multiple model runs. Therefore, we set $G=1$.

\section{c. Model configurations}

Since fractal calculations require better dipole resolution and Euclidean calculations require many separate runs, we perform separate simulations for each case. For Euclidean only runs, we build 2000 aggregates whereas for fractal runs we build only 20 . We can calculate mean fractal quantities (generalized fractal dimension and lacunarity) for each model run and then average over each simulation. In this sense, each model run represents a different realization of aggregates with $N_{\text {mon }}$ number of monomers for given initial conditions. For our tests, we have a fixed dipole size of 0.01 (arbitrary units). We found in our initial tests that only three $l$ grid scales of $N_{\text {grid }}=4,8$, and 16 (see Fig. 4) were necessarily to maintain consistent fractal results throughout evolution. Grid scales that are too close to the aggregate size (i.e., $N_{\text {grid }}=2$ ) or too close to the dipole size (i.e., $N_{\text {grid }}=32$ and 64) will not capture the appropriate fractal scaling.

Our Monte Carlo aggregate tests build upon those performed by Westbrook et al. (2004a,b) and Schmitt and Heymsfield (2010). We repeat the tests of Schmitt and Heymsfield (2010) for $\min \left(\varphi_{\operatorname{mon}}, \varphi_{\operatorname{mon}}^{-1}\right)=1.0$ and 0.5 but we also perform tests where $\min \left(\varphi_{\operatorname{mon}}, \varphi_{\text {mon }}^{-1}\right)=$ 0.1 and 0.05 instead of $\min \left(\varphi_{\text {mon }}, \varphi_{\text {mon }}^{-1}\right)=0.2$ to explore more of the aggregate parameter space. Here, the min function refers to the minimum value of $c / a$ and $a / c$. This convention signifies that each set of oblate and prolate monomer runs has the same ratio of minor to major axis lengths. We also aggregate monomers of different aspect ratios by performing two additional sets of simulations of fixed monomer volume or fixed maximum dimension. These two additional tests allow us to explore how different monomer combinations affect the aggregate evolution. When calculating the evolution of ellipsoid quantities for a fixed monomer volume, we use a total of about 520 dipoles to represent each monomer whereas for fractal simulations we use a total of about 4200 dipoles. The number of dipoles for each monomer is slightly variable when reorienting each monomer during aggregation. This is because dipole indices are given in terms of integer values, which means some dipoles are rounded to the same location. For runs where we hold the monomer $a$ axis constant, we increase the number of dipoles by roughly twice that of the other tests. This increase in resolution is necessary since a fixed dipole size severely limits the number of dipoles that can make up eccentric particles (particularly prolate spheroids). Additional tests with higher resolution (not shown) showed little difference in fractal and Euclidean quantities. For aggregating particles of different orientation we use two extreme cases: random and horizontal. Random orientation of Euler angles follows that of Jiang et al. (2017). Horizontal orientations are the same as random orientations with the exception that the second rotation is held at zero which keeps the maximum dimension always along the horizontal $x-y$ plane. For multifractal runs, we average $D_{q}$ over 20 orientations for each aggregate realization from $-10 \leq q \leq+10$ to determine multifractal behavior.

\section{Results}

\section{a. Monomers of the same habit and aspect ratio}

Figure 5 shows the evolution of aggregate ellipsoid aspect ratios for different initial monomer aspect ratios and $N_{\text {mon }}=2,3,10,50$, and 100 . The symmetry of spherical monomers restrict aggregates with $N_{\text {mon }}=2$ to always produces prolate spheroids with $\varphi_{b a}=\varphi_{c a}=0.5$. However, as more and more monomers are added, the ellipsoid bivariate aspect ratio distribution shifts toward aspect ratios of unity. When $N_{\text {mon }}=100$, the dominant shape is close to prolate spheroids with two separate modes $\left(\varphi_{b a}=\varphi_{c a} \approx 0.4\right.$ and $\left.\varphi_{b a}=\varphi_{c a} \approx 0.8\right)$. For oblate spheroids (plates) and prolate spheroids (columns) with aspect ratios of $\min \left(\varphi_{\text {mon }}, \varphi_{\text {mon }}^{-1}\right)=0.5$, the ellipsoid evolution from $N_{\text {mon }}=3$ to $N_{\text {mon }}=100$ is nearly identical to that of spheres. The only monomer type that deviates from this evolution is that of $\varphi_{\text {mon }}=10$ prolate spheroids. For this case at $N_{\text {mon }}=2$, the distribution mode is close to $\varphi_{b a} \approx \varphi_{c a} \approx 0.2$ but much of the ellipsoid parameter space is available as potential aggregates. By $N_{\text {mon }}=10$, all cases have distributions that look identical. However, for $N_{\text {mon }}=50$ and 100 , the $\varphi_{\text {mon }}=10$ case has its mode concentrated more toward unity and has much less overall spread in values. The aggregate ellipsoid $a$ axis scaling, compared to the $a$-axis length of each monomer, is shown in Fig. 6 for the model runs shown from Fig. 5. For all cases, the ratio of the aggregate ellipsoid $a$-axis length to monomer $a$-axis length increases roughly as a power-law with $N_{\text {mon }}$. This scaling is most pronounced for spheres and least pronounced with thin columns. The spread in ellipsoid $a$-axis lengths is largest for spheres but smallest for thin columns. The combination 


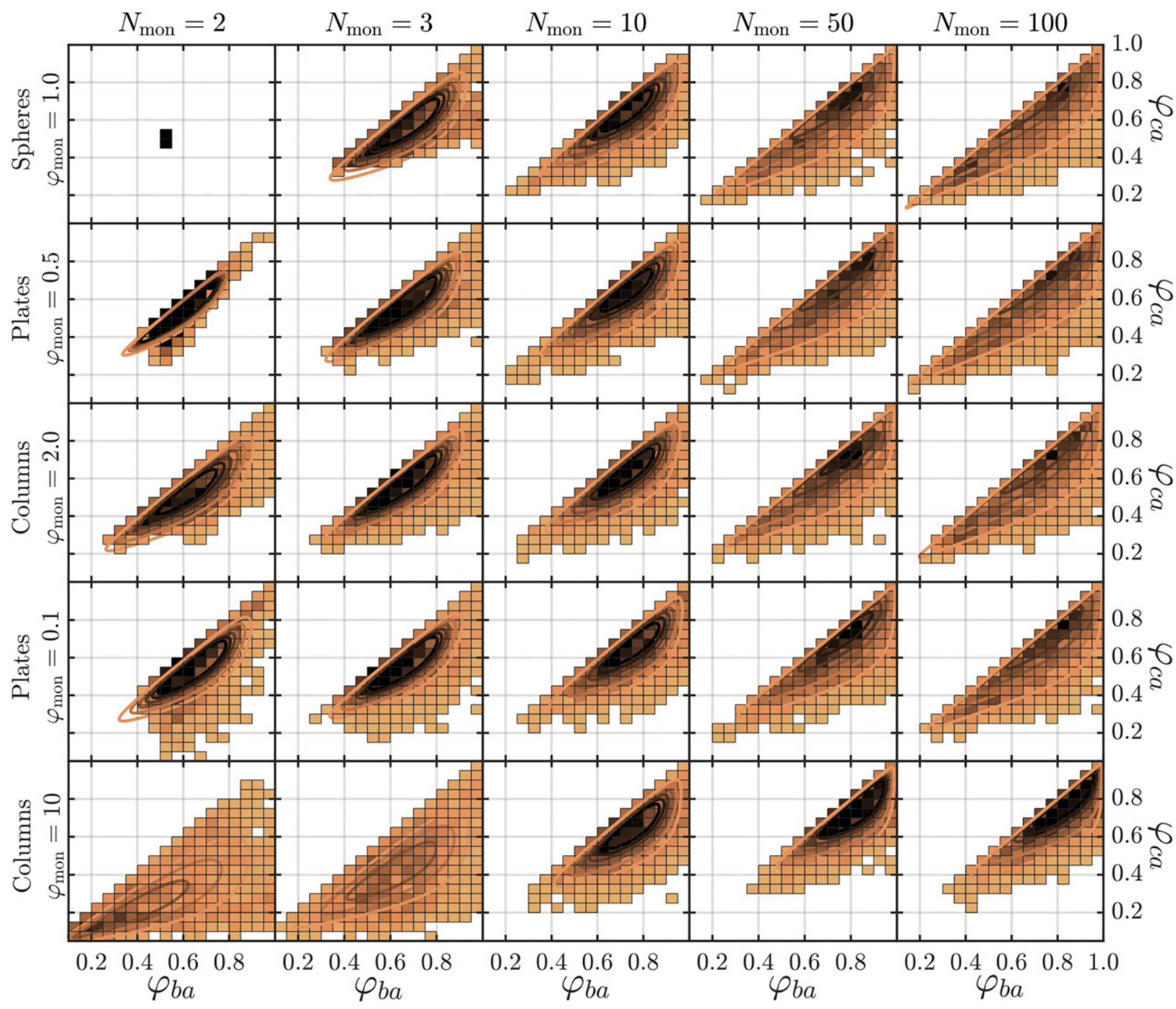

$0 \quad 2$
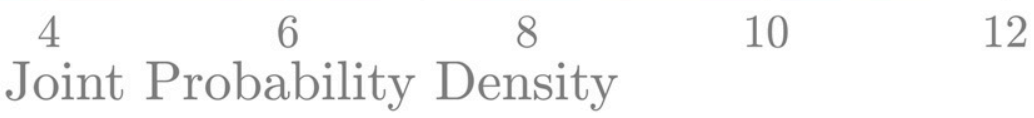

FIG. 5. Evolution of ellipsoid distributions for different monomer aspect ratios for Monte Carlo simulations (joint histograms) and mathematical model (contours). Contours range from 2 to 12 in increments of 2 .

of Figs. 5 and 6 illustrates the entire evolution of the ellipsoid geometry for each case. As a result, although the shape evolution is consistent between plate and sphere aggregates, the decreased $a$-axis lengths for plate aggregates suggests that these ellipsoids are scaled in a different way than sphere aggregates.

Figure 7 shows a comparison of the fractal evolution alongside the Euclidean evolution of shape and density. The fractal dimension of aggregates composed of spherical monomers behave very much like Westbrook et al. (2004b) and Schmitt and Heymsfield (2010), where the mean fractal dimension for low numbers of monomers are higher than those with more monomers. After a couple dozen monomers, the mean fractal dimension is essentially constant with $D_{f} \approx 2.2$. However, for eccentric plates and columns, the mean fractal dimension monotonically increases throughout evolution. Aggregates composed of thin columns $\left(\varphi_{\text {mon }}=10\right)$ exhibit significantly lower fractal dimensions. This behavior is even more exaggerated when $\varphi_{\text {mon }}=20$ whereas the fractal dimension for plates with $\varphi_{\text {mon }}=0.05$ is essentially unchanged from that of $\varphi_{\text {mon }}=0.1$. The limiting behavior of mean 


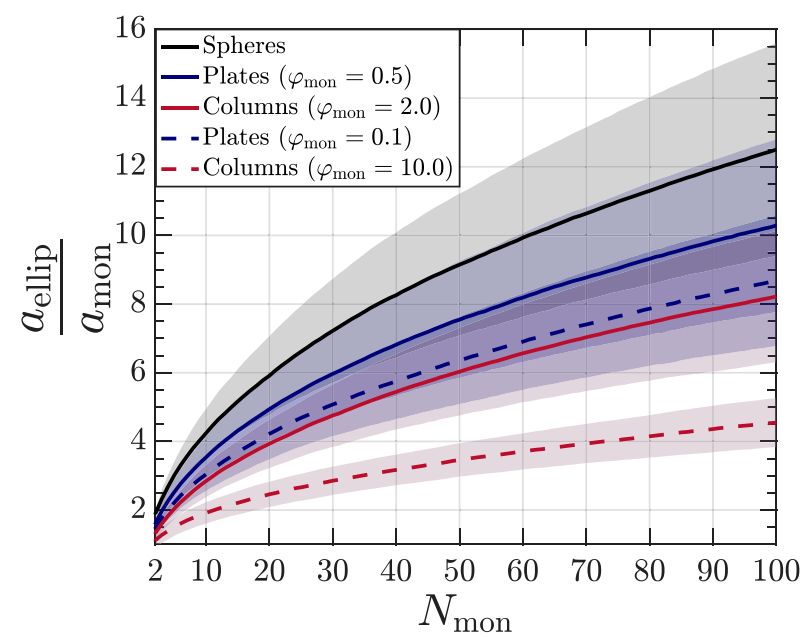

FIG. 6. Evolution of the ratio between the aggregate ellipsoid $a$ axis and the monomer $a$ axis as a function of $N_{\text {mon }}$ for the Monte Carlo simulations shown in Fig. 5. Lines represent mean values over all 2000 aggregate realizations and shaded regions represent \pm 1 standard deviation about each mean value.

lacunarity seems to mirror that of the fractal dimension for each monomer type. Lacunarity for plate aggregates increases (increasing porosity) until about $N_{\text {mon }}=20$ where it becomes approximately constant with $\bar{\Lambda} \approx 0.95$.
Sphere aggregates exhibit a more prolonged lacunarity evolution that tends toward that of plate aggregates. Aggregates composed of thin columns, however, have lacunarity that is higher than plates and spheres but tends to decrease slightly after about 25 monomers.

The evolution of the mean ellipsoid shapes and densities (as volume fractions of total aggregate volume) are shown in the center and right columns of Fig. 7. For plates and spheres, $\bar{\varphi}_{b a}$ and $\bar{\varphi}_{c a}$ (top center) increases until $N_{\text {mon }} \approx 10$ and then slowly decreases. This behavior is also seen for the first product moment (top right). Overall, aggregates composed of plates, regardless of monomer aspect ratio, exhibit a consistent ellipsoid shape evolution. Column aggregates on the other hand have mean aspect ratio quantities that increase until $N_{\text {mon }} \approx 50$. This extended evolution for thin column aggregates results in best-fit ellipsoids that end up becoming more spherical than either plates or spheres. Volume fractions (densities) of aggregates are in general decreasing (increasing lacunarity) with number of monomers (increase in size). However, for all model runs, the use of ellipsoids instead of spheres acts to substantially decrease the spread of potential density values. This decrease in spread is due to the better representation of aggregate shape provided by ellipsoids.
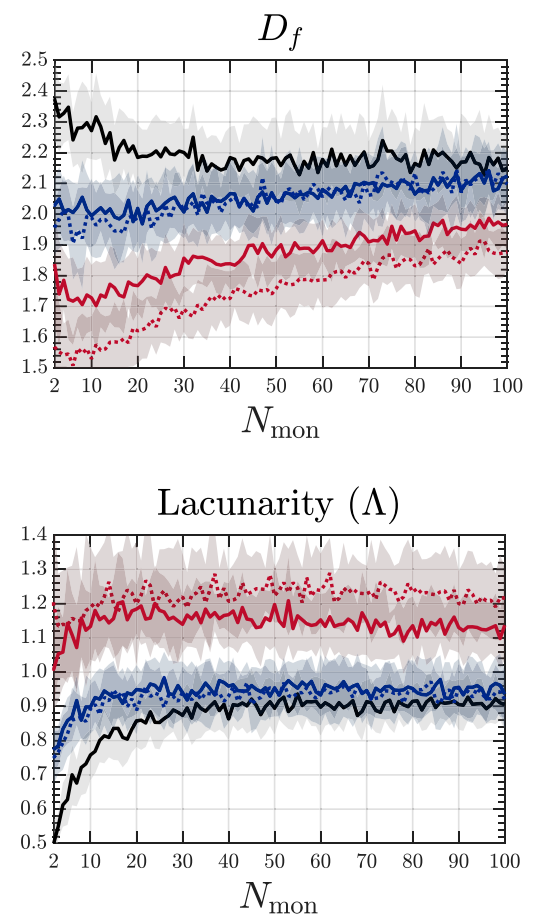
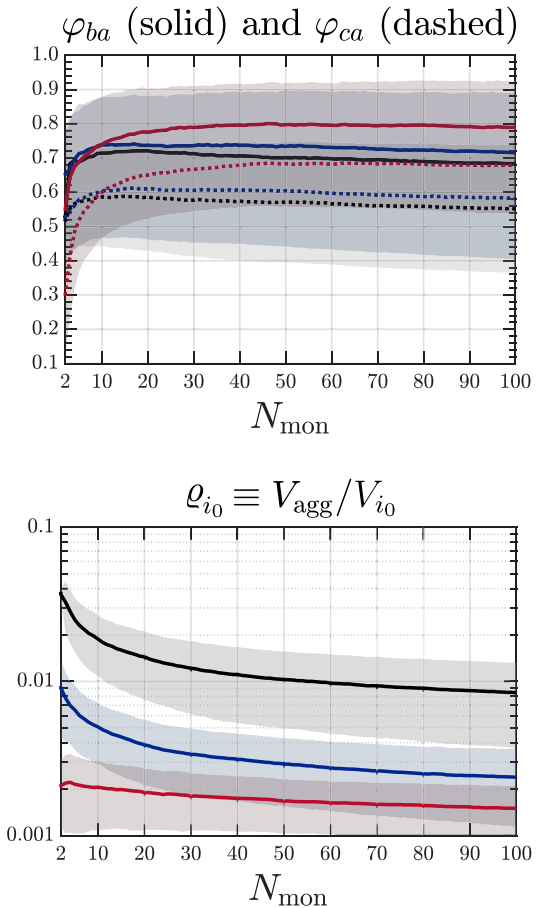
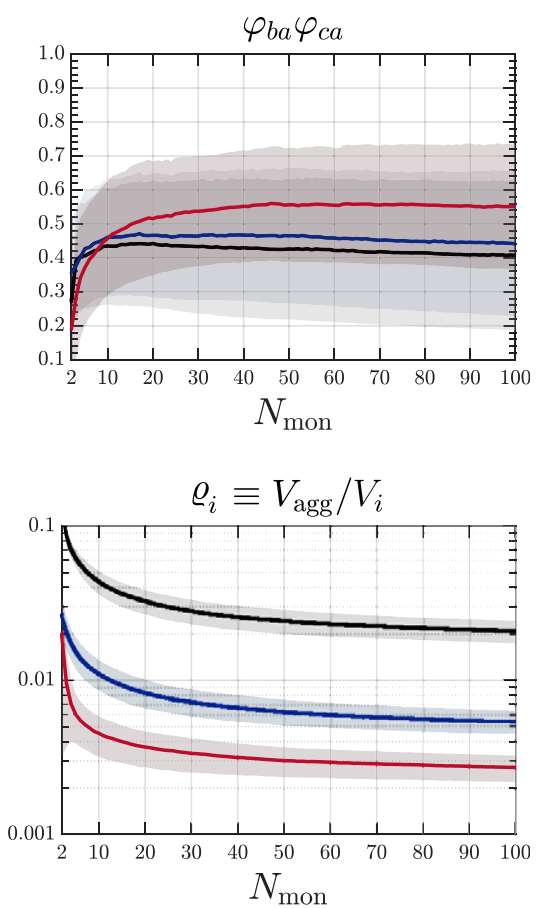

FIG. 7. Fractal and Euclidean evolution of identical monomer aggregates under random orientations. Red lines correspond to prolate spheroid monomers, blue lines correspond to oblate spheroid monomers, and black lines correspond to sphere monomers. (left) Fractal quantities were calculated over 20 model runs whereas (middle),(right) Euclidean quantities were calculated over 2000 model runs. Lines represent the mean values over all runs whereas shaded regions represent \pm 1 standard deviation. Dotted lines for fractal quantities represent cases where $\min \left(\varphi_{\text {mon }}, \varphi_{\text {mon }}^{-1}\right)=0.05$. 

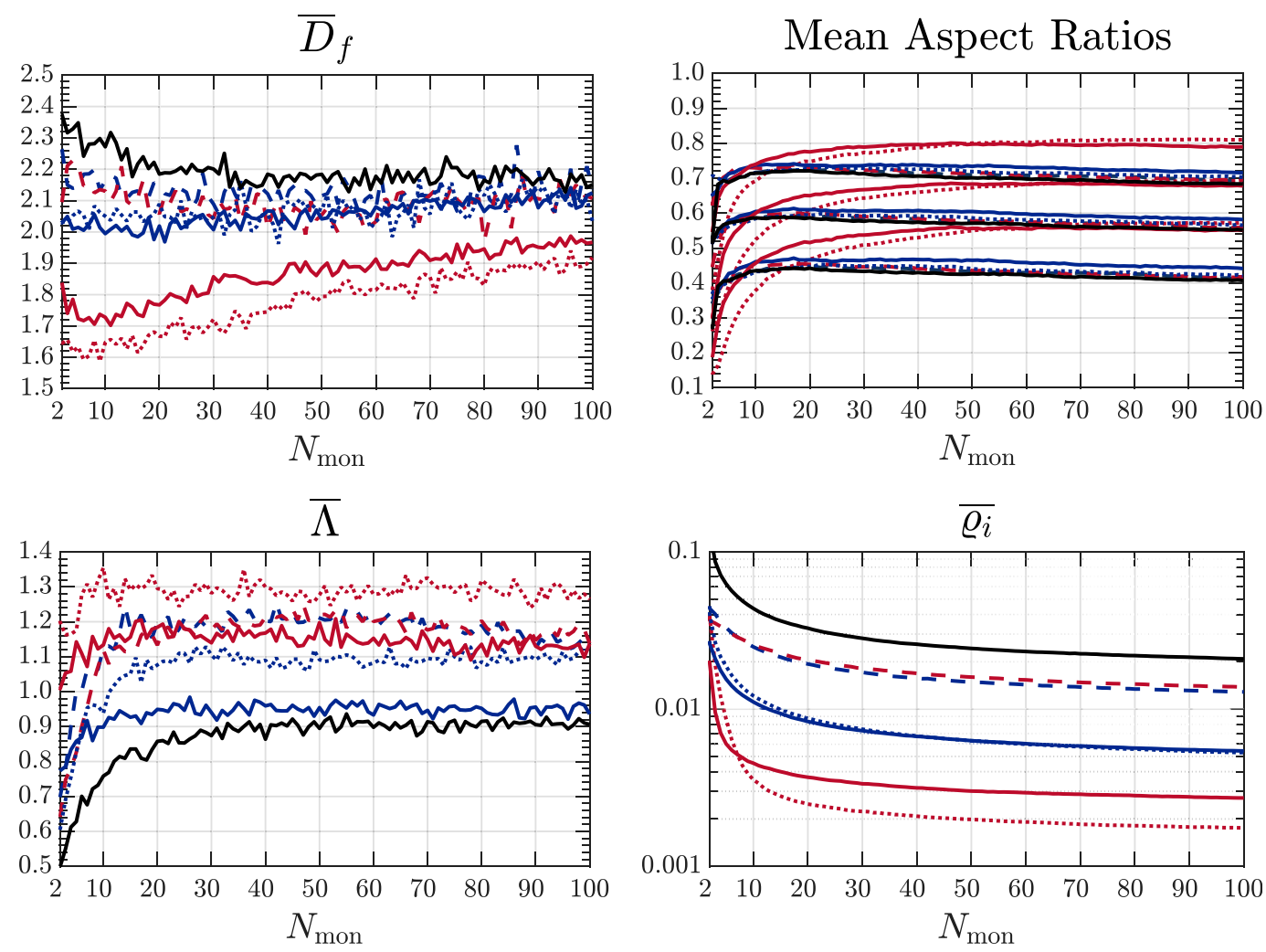

FIG. 8. Evolution of the mean ellipsoid and fractal properties for mixed aspect ratio cases of plate aggregates (blue), column aggregates (red), and spheres (black). Solid lines correspond to results for $\min \left(\varphi_{\operatorname{mon}}, \varphi_{\text {mon }}^{-1}\right)=0.1$ from Fig. 7 and mixed cases represent randomly selected aspect ratios of either 0.1 or 0.5 . Dashed lines represent mixed aspect ratios with same monomer $a$-axis length whereas dotted lines represent mixed aspect ratios with the same monomer volume. Mean aspect ratios are from top to bottom: $\bar{\varphi}_{b a}, \bar{\varphi}_{c a}$, and $\left\langle\varphi_{b a} \varphi_{c a}\right\rangle$.

Different aggregate realizations do not substantially increase the spread of various density values for ellipsoids. Moreover, although the mean aspect ratio quantities for thin plates are very similar to that of spheres, the density values are much lower. This discrepancy in evolution suggests that a single density relationship for aggregates is not sufficient to fully capture ellipsoidal aggregate geometry for all types of aggregates.

\section{b. Monomers of the same habit but different aspect ratios}

Figure 8 shows a comparison of mean Euclidean and fractal quantities for cases where we randomly $\operatorname{select} \min \left(\varphi_{\text {mon }}, \varphi_{\text {mon }}^{-1}\right)=0.1$ or 0.5 . For comparison, the $\min \left(\varphi_{\text {mon }}, \varphi_{\text {mon }}^{-1}\right)=0.1$ and sphere monomer cases are included as well. The sphere monomer case is used as a proxy for $\min \left(\varphi_{\text {mon }}, \varphi_{\text {mon }}^{-1}\right)=0.5$ since the fractal and Euclidean properties are nearly identical.

For monomers of the same $a$-axis length but different aspect ratios, both fractal and Euclidean properties are very similar for each habit. For both plate and column aggregates, these mean fractal dimensions are roughly halfway between that of $\varphi_{\text {mon }}=0.1$ plates and $\varphi_{\text {mon }}=$ 0.5 (or spheres) for $N_{\text {mon }}<35$. However, these mixed cases become nearly identical to that of $\varphi_{\text {mon }}=0.1$ plate aggregates for $N_{\text {mon }} \geq 35$. The mean lacunarity for these cases is always higher than that of $\varphi_{\text {mon }}=0.1$ plate aggregates and behaves more like column aggregates with $\varphi_{\text {mon }}=10$. For aggregates composed of equal volume but mixed aspect ratio monomers, the fractal behavior is similar to that of $\min \left(\varphi_{\text {mon }}, \varphi_{\text {mon }}^{-1}\right)=0.1$. For instance, the behavior of $\bar{D}_{f}$ follows that of $\varphi_{\text {mon }}=10$ but lower in value whereas for plate aggregates the $\bar{D}_{f}$ evolution is very close to that of $\varphi_{\text {mon }}=0.1$. Unlike $\bar{D}_{f}$, the mean lacunarity for aggregates composed of mixed aspect ratio monomers is substantially higher than the $\varphi_{\text {mon }}=$ 0.1 case. The fact that $\bar{D}_{f}$ does not vary much for plate aggregates whereas $\bar{\Lambda}$ does suggests that $\alpha_{m}$ and $\beta_{m}$ are not necessarily related in a unique way; the same $\beta_{m}\left(D_{f}\right)$ can correspond to multiple values of $\alpha_{m}(\Lambda)$. This lack of consistency is particularly a problem if an assumed initial aggregate size and a fixed $\beta_{m}$ are both used to solve for $\alpha_{m}$ using an extrapolation approach similar to Schmitt and Heymsfield (2010). 

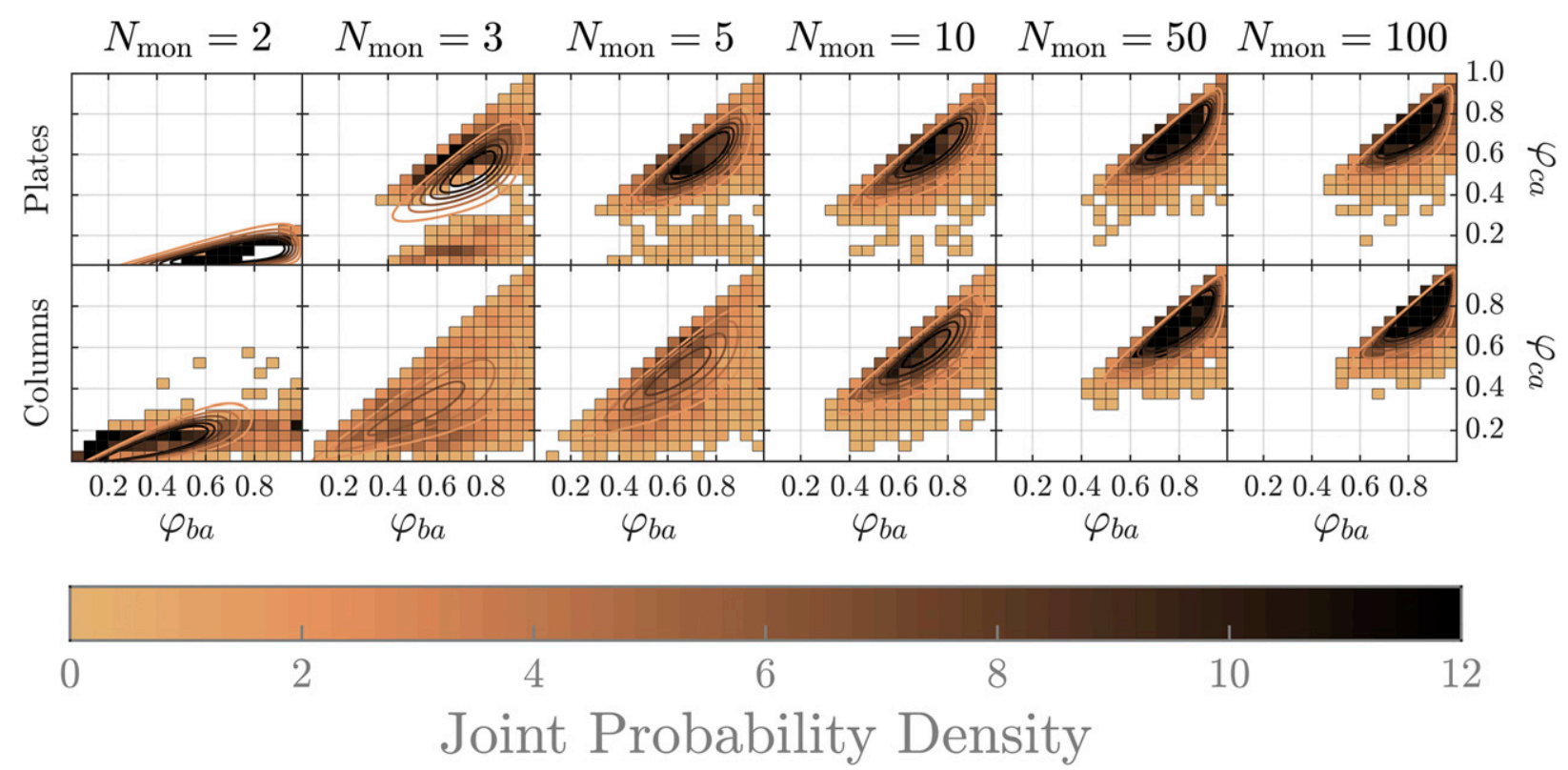

FIG. 9. As in Fig. 5, but for horizontal particle orientations and $\min \left(\varphi_{\operatorname{mon}}, \varphi_{\operatorname{mon}}^{-1}\right)=0.1$.

Euclidean estimates of aspect ratios and densities generally mirror the limiting behavior of their fractal counterparts. For instance, mean aspect ratio quantities for plate aggregates are within a few percent of $\varphi_{\text {mon }}=$ 0.1 aggregates and sphere aggregates regardless of different monomer aspect ratio combinations. Likewise, column aggregate mean aspect ratios follow a similar limiting behavior of $\varphi_{\mathrm{mon}}=10$. However, the ellipsoid volume fractions show some important differences. Perhaps most surprising is that while the ellipsoid volume fractions for plate monomers of equal volume, but mixed aspect ratios produce aggregates that are similar to $\varphi_{\text {mon }}=0.1$ aggregates, the lacunarity is much higher. Therefore, it seems that the analogy between $\Lambda$ and $\rho_{i}$ is rather imperfect. Nevertheless, aggregates composed of the same maximum dimension but different aspect ratios seem to behave in a consistent way regardless of habit. This consistency is possibly the result of the more voluminous monomers contributing more to the overall aggregate geometry. However, the fact that $\bar{\Lambda}$ and $\bar{\rho}_{i}$ are not necessarily between their extreme aspect ratio cases suggests that aggregate characteristics depend nonlinearly on monomer properties. Mixed habit tests (not shown) also illustrate similar behavior although the exact mathematical relationship between the geometry of these aggregates and their monomers is not clear.

\section{c. Effect of orientations}

Figure 9 shows the ellipsoid evolution of aggregates for plate and column monomers with $\min \left(\varphi_{\text {mon }}, \varphi_{\text {mon }}^{-1}\right)=0.1$ when particles are assumed to fall with their maximum dimensions in the horizontal. For both cases, the early ellipsoidal evolution $2 \leq N_{\text {mon }} \leq 5$ is much more restricted than that of random orientations (Fig. 5). For plate monomers, only a few ellipsoid aspect ratio pairs are allowed; aggregates are either stacked (i.e., an oblate spheroid with $\varphi=2 \varphi_{\text {mon }}$ ), touching edges (i.e., an ellipsoid with $\varphi_{b a}=0.5$ and $\varphi_{c a}=\varphi_{\text {mon }}$ ), or some configuration in between. Intermediate cases therefore correspond to a roughly diagonal region within the ellipsoid parameter space. For column aggregates, horizontal orientations essentially nearly eliminate the top half of the ellipsoid aspect ratio parameter space from Fig. 5. For horizontal orientations, column aggregates are either stuck more or less from end to end (i.e., $\varphi_{b a} \approx \varphi_{c a} \approx \varphi_{\text {mon }} / 2$ ), stuck more or less in a crossed " $\mathrm{X}$ " configuration (i.e., $\varphi_{b a} \approx 1.0$ and $\varphi_{c a} \approx$ $\left.2 \varphi_{\text {mon }}\right)$, or a configuration somewhere in between. Intermediate cases therefore correspond to a roughly horizontal region within the ellipsoid aspect ratio parameter space.

When $N_{\text {mon }}=3$, plate aggregates correspond to two separate regions of the aspect ratio parameter space whereas column aggregates with $N_{\text {mon }}=3$ look much like that of random orientations but with a peak at lower aspect ratios. By $N_{\text {mon }}=10$, both cases are very similar to that of Fig. 5. However, unlike their random orientation counterparts in Fig. 5, horizontal orientations yield a nearly identical evolution for $N_{\text {mon }}=10$ to $N_{\text {mon }}=100$ with aspect ratios that peak closer to unity.

\section{d. Aggregate-aggregate collection}

All results so far represent aggregates that have been built monomer-by-monomer. However, it is also expected 
that aggregates should also collect with other aggregates. It is quite possible that aggregates formed monomer-bymonomer have different geometries than those formed aggregate-by-aggregate since Schmitt and Heymsfield (2010) found that aggregate-aggregate collection was required to explain inconsistencies between modeled and observed 2D area ratios. Here, we use the approach of Schmitt and Heymsfield (2010) to test aggregateaggregate collection on the resulting ellipsoid distribution. To do this, we randomly generate and store aggregates with 2-5 monomers. We then randomly select these aggregates and follow the same approach from section 3 assuming random orientations of aggregates. Aggregates are collected together 20 times such that the largest aggregates of each model run have $40 \leq N_{\text {mon }} \leq 100$.

The resulting ellipsoid distribution for $\varphi_{\text {mon }}=0.1$ are shown in Fig. 10. Because each collection event yields a variable number of monomers for each aggregate, we show the distribution resulting from all collection events. This final distribution takes the same form of the other model runs (Fig. 5) where the asymmetric bivariate form is consistent the MASC observations (Fig. 2) but has aspect ratios that are slightly higher. Overall, the mean aspect ratios shown in Fig. 10 are quite close to those shown in $\varphi_{\text {mon }}=0.1$ for $N_{\text {mon }} \approx 10$. Therefore, the resulting ellipsoid distribution for aggregate-aggregate collection does not seem to deviate substantially from monomer-aggregate collection.

\section{e. Multifractal evolution}

One potential way to determine when aggregates become fully developed is to track when the multifractal spectrum stops changing. An unchanging spectrum signifies that regardless of how the aggregate constituents are perturbed, the overall fractal behavior stays the same. For our purposes, this suggests that visually different aggregates have physical properties that are essentially the same. Figure 11 shows how the multifractal spectrum (which in this context is $q$ vs $D_{q}$ ) evolves for different $N_{\text {mon }}$ and for different monomer types. Note that each spectrum generally monotonically increases in $q$ which is consistent with van Opheusden et al. (1996, their Fig. 9) who have calculated similar spectra for their snowflake aggregates. The origin of this increasing spectrum is thoroughly discussed in van Opheusden et al. (1996) and van Opheusden (1998) and seems to be the result of building aggregates whose monomers exhibit an excluded volume effect. Diffusion-limited aggregates like dendrites, by contrast, have decreasing multifractal spectra (cf. Vicsek et al. 1990; Wolf 1996).

One interesting aspect about Fig. 11 is that negative multifractal moments are nearly identical throughout

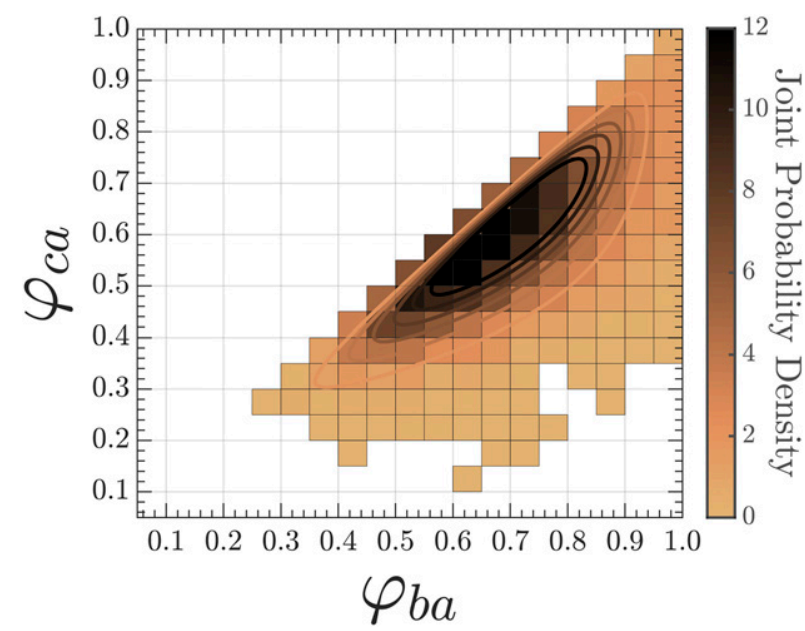

FIG. 10. As in Fig. 5, but for aggregate-aggregate collection following the approach of Schmitt and Heymsfield (2010).

evolution and asymptote toward the same value as $q \rightarrow-\infty$. It is not clear why this behavior is consistent for each case although it could be due to the annealed averaging of the partition function for very large and very small numbers (see Wolf 1996). Therefore, we restrict our analysis to positive moments only. In general, the multifractal spectrum for sphere monomers evolves slower than the other cases. The spectrum monotonically increases for each $N_{\text {mon }}$ aggregate. However, as aggregates collect more monomers, $D_{q}$ corresponding to positive moments flattens out. This flattening out effect is much more pronounced for the planar and columnar habits and does not appear to be a result of the choice of grid scales or dipole resolutions. As discussed in van Opheusden et al. (1996), the anomalous increasing multifractal spectrum acts to swap the interpretation of $D_{0}$ and $D_{2}$. The fact that positive moments of the multifractal dimensions are generally flat is therefore reassuring since this increasing spectrum suggests the use of $D_{2}$ instead of $D_{0}$ as a proxy for $\beta_{m}$. Flatter multifractal spectra signify more monofractal behavior. Therefore, these spectra suggest that the early stage aggregates are better represented as multifractals whereas mid to late stage aggregates can be thought of as monofractals. Other numerical studies, such as Warren and Ball (1989), have described these early aggregation multifractal regimes as an "approach to scaling." The prolonged approach to fractal scaling for thin, prolate monomers as shown in Figs. 7, 8, and 11 is qualitatively consistent with aggregates of "infinitely thin rods" shown in Fig. 3 of Westbrook et al. (2004a). For these aggregates, Westbrook et al. (2004a) found that almost 100 monomers were required for the aggregate mass-dimensional relationship to assume the form of Eq. (1) for a constant fractal dimension. 


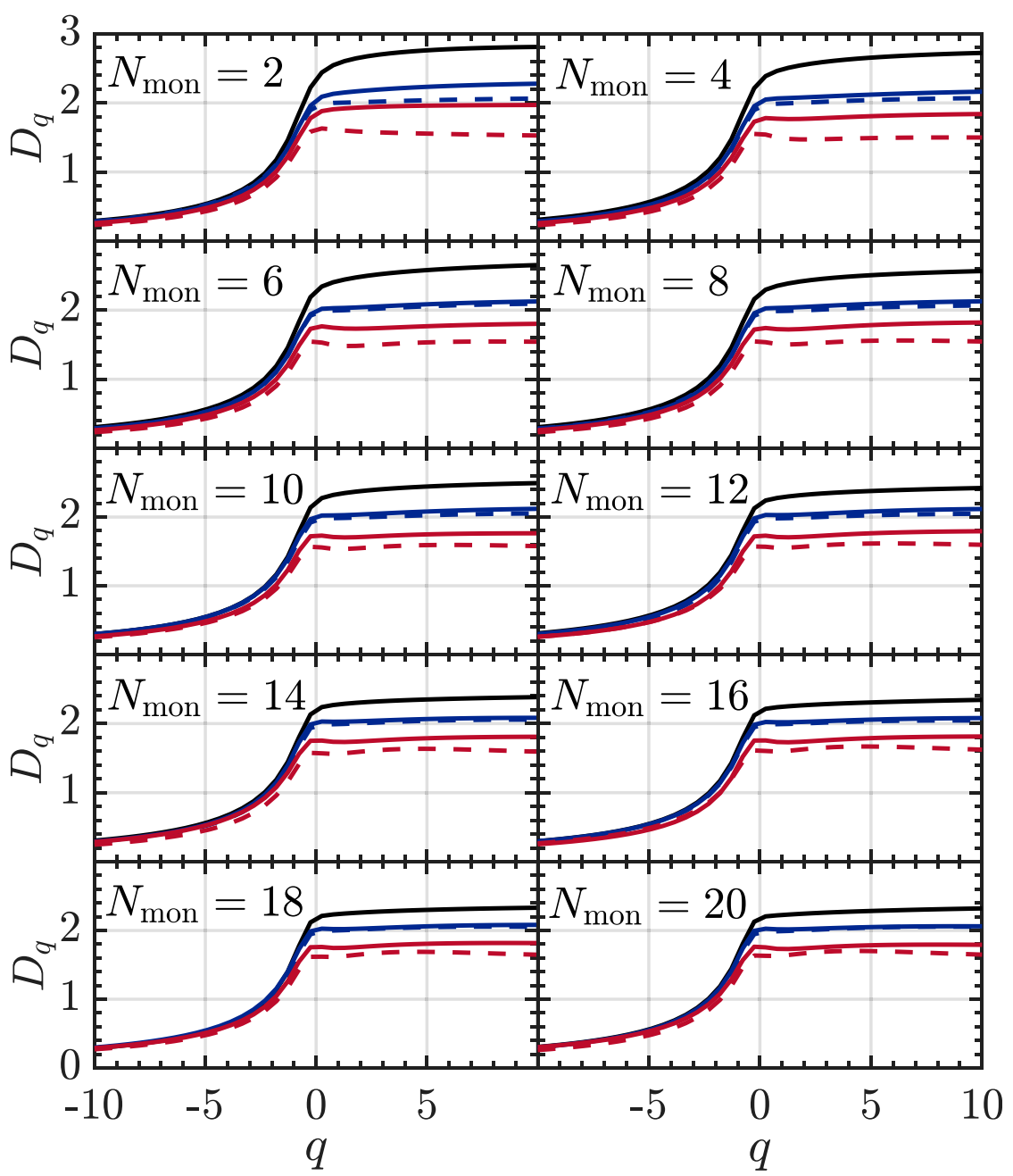

FIG. 11. Multifractal evolution of aggregates for spheres (black line), oblate spheroids (blue lines), and prolate spheroids (red lines). Solid lines represent aspect ratios of 0.1 and 10 and dashed lines represent aspect ratios of 0.05 and 20. Each panel represents the average spectrum over 20 simulation runs and $G=20$ orientations.

Conversely, aggregates composed of more spherical monomers from Fig. 2 in Westbrook et al. (2006) converge to the fractal scaling much more rapidly with fewer monomers than in Westbrook et al. (2004a). This is also qualitatively consistent with our results.

\section{Discussion}

\section{a. A possible explanation for universal ellipsoid distributions}

The persistent bivariate pattern of the Monte Carlo aggregate best-fit ellipsoid distributions (Figs. 5 and 9) and that of observations (Fig. 2) suggests some sort of universality of the aggregation process that exists regardless of monomer properties. Oblate spheroids are surprisingly not preferred at any time for any of our tests. Therefore, our consistent asymmetric, triaxial configurations counter some claims that early stage aggregates are approximately oblate (Moisseev et al. 2015). Even for the aggregation of horizontally oriented planar crystals, the best-fit ellipsoid distribution produces predominately triaxial ellipsoids that are rarely oblate. One natural question to ask is why and how does this asymmetry manifest?

Here, we propose a possible hypothesis. Consider the case when $N_{\text {mon }}=2$. Since monomers are not allowed to occupy the same space (volume exclusion), any configuration is naturally asymmetric in $(x, y, z)$. If there is no preference for any particular lattice site (which is possibly the case if the major dimension is randomly oriented in the $x-y$ plane), then the next aggregation event $\left(N_{\text {mon }}=3\right)$ will have a higher likelihood of occurring 
along the major dimension ( $a$ axis) rather than either minor dimensions ( $b$ or $c$ axes). This is simply because there are more lattice sites along the major dimension than the two minor dimensions. Each additional monomer contributes less and less to the overall aggregate size. Therefore, in order to produce an oblate or spherical aggregate, aggregation events must occur with unequal probability in the $x-y$ plane to counteract the initial asymmetric aggregate shape. As a result, the probability of extending the major ellipsoid $a$-axis length is naturally larger than the other two axes; there are simply more attachment sites that are projected along the $a$ axis versus the other axes. The ellipsoid evolution therefore could possibly be thought of as a Dirichlet-type process like the Chinese restaurant process (Blei et al. 2003). The Chinese restaurant process is a type of stochastic clustering process whereby cluster locations (imagined as tables in a Chinese restaurant) will incorporate additional integers (imagined as customers) according to probabilities that are weighted based on the current occupancy of each table. This conceptual structure generates a distribution on partitions of integers. The consistent bivariate aspect ratio beta distribution form suggests that the aggregation process, like the Chinese restaurant process, is exchangeable: The order in which monomers of various orientations aggregate does not affect the final distribution. Viewing aggregation as a Dirichlet process could also explain why the marginal aspect ratio distributions are so well represented as beta distributions since the multivariate Dirichlet distribution is essentially a multidimensional beta distribution. Future aggregation work could explore this possibility by using a Dirichlet Markov chain Monte Carlo (MCMC) model or a Gaussian mixture model.

MCMC or Gaussian mixture models can help describe the evolution of the aspect ratio distributions but the evolution of each mean aspect ratio could possibly result from simple mathematical scaling relationships. Warren and Ball (1989) studied the aggregation problem using 3D ellipsoid fits and described the Euclidean and fractal approach to scaling based on such simple mathematical relationships. These scaling relationships stem from mapping the axis lengths of the monomer ellipsoids to the aggregate ellipsoids, thereby yielding a fractallike scaling for each axis length. From the transformation matrix of this mapping, Warren and Ball (1989) was able to estimate fractal dimensions of the aggregates from the largest eigenvalue and correction factors based on the second largest eigenvalue. For their study, Warren and Ball (1989) used three different methods for generating each aggregate. The first method, a model configuration approach, considered the subset of configurations where the shortest axis of one ellipsoid abuts against the longest axis of another. The second method averages the configurations of the first where the axis spans cross over (i.e., the longest axis of the aggregate is constructed based on the minor axes of each monomer). The third method is a computer simulation where aggregation occurs on a cubic lattice grid space.

Table 1 shows a comparison among these results, the results from the current paper and results from Schmitt and Heymsfield (2010) for both mean ellipsoid aspect ratios and fractal dimensions where appropriate. Overall, both sets of ellipsoid fitting from Warren and Ball (1989) and this paper exhibit the same type of asymmetric scaling among each aspect ratio but are higher than the MASC observations. The scaling approach presented by Warren and Ball (1989) provides insight into how these differences can manifest. Some potential scaling relationships are also shown in Table 1 as well as their fixed points (in terms of their equilibrium mean aspect ratios) and largest eigenvalue (fractal dimension) of their transformation matrices. Each ellipsoid axis length (indicated by primes) is assumed to scale linearly according to the axis lengths of each monomer. Fixed points of axis lengths (or alternatively, aspect ratios) indicate that the aggregate ellipsoid is a scaled up version of their monomers. Iterating the first scaling relationship shown in Table 1 yields mean ellipsoid aspect ratios that are quite close to our randomly oriented, sphere monomer Monte Carlo simulations. While the fractal dimension from the transformation matrix is larger than that of our estimates, this fractal dimension is within 0.02 of Schmitt and Heymsfield (2010). The second scaling relationship in Table 1 represents that of the first scaling but with a slightly different weighting for the $a$ axis. This less steep maximum axis scaling is consistent with that of thin column aggregates as shown in Fig. 6 compared to sphere aggregates. The resulting mean ellipsoid aspect ratios are naturally higher than that of sphere aggregates and are consistent with those of column aggregates (Figs. 7 and 8). A hint of this different scaling regime can be seen in Figs. 7 and 8 where $\bar{\varphi}_{c a}$ for thin column aggregates tends toward that of $\bar{\varphi}_{b a}$ for sphere aggregates. The associated decrease in fractal dimensions from this second scaling regime is also quantitatively consistent with that of the Monte Carlo simulations. The third scaling relationship in Table 1 is very close to the model configuration methodology of Warren and Ball (1989) where $\bar{\varphi}_{b a}$ and $\bar{\varphi}_{c b}$ are almost identical and higher than $\bar{\varphi}_{c a}$. However, the fractal dimension from the model configuration simulation is quite a bit lower than that from the scaling relationship. Finally, the fourth scaling regime is one scaling example that can explain the MASC observations. Future work could investigate these 
TABLE 1. Comparison of mean quantities from Monte Carlo simulations, MASC data, Schmitt and Heymsfield (2010), and Warren and Ball (1989). Mean aspect ratios are reported for $N_{\text {mon }}=100$ for Monte Carlo simulations and $N_{\text {mon }}=1000$ for the data from Warren and Ball (1989) (their Table 2 and Fig. 5). Mean fractal dimensions are calculated for $25 \leq N_{\text {mon }} \leq 100$ for Schmitt and Heymsfield (2010) (their Table 1). Boldface fonts highlight the different possible relationships between scaling regime and modeling/observation results as discussed in section 5 a.

\begin{tabular}{|c|c|c|c|c|}
\hline Model/observation & $\bar{\varphi}_{b a}$ & $\bar{\varphi}_{c a}$ & $\bar{\varphi}_{c b}$ & $\bar{D}_{f}$ \\
\hline MASC North Slope of Alaska (NSA) & 0.6160 & 0.4186 & 0.6904 & - \\
\hline MASC Alta, Utah (Alta) & 0.6269 & 0.4397 & 0.7068 & - \\
\hline Warren and Ball (1989) model configuration & 0.6837 & 0.4651 & 0.6803 & 1.81 \\
\hline Warren and Ball (1989) averaged model & 0.6650 & 0.4854 & 0.7299 & 1.90 \\
\hline Warren and Ball (1989) computer simulation & 0.6540 & 0.4739 & 0.7246 & 1.97 \\
\hline Schmitt and Heymsfield (2010) spheres & - & - & - & 2.23 \\
\hline Schmitt and Heymsfield (2010) plates $\left(\varphi_{\text {mon }}=0.5\right)$ & - & - & - & 2.22 \\
\hline Schmitt and Heymsfield $(2010)$ columns $\left(\varphi_{\text {mon }}=2.0\right)$ & - & - & - & 2.17 \\
\hline MC spheres & 0.6837 & 0.5511 & 0.8056 & 2.15 \\
\hline MC plates $\left(\varphi_{\text {mon }}=0.5\right)$ & 0.6959 & 0.5635 & 0.8069 & 2.15 \\
\hline $\operatorname{MC}$ columns $\left(\varphi_{\text {mon }}=2.0\right)$ & 0.6946 & 0.5600 & 0.8061 & 2.22 \\
\hline MC plates $\left(\varphi_{\text {mon }}=0.1\right)$ & 0.7154 & 0.5814 & 0.8116 & 2.12 \\
\hline $\mathrm{MC}$ columns $\left(\varphi_{\mathrm{mon}}=10.0\right)$ & 0.7898 & 0.6779 & 0.8589 & 1.97 \\
\hline MC plates $\left(\varphi_{\text {mon }}=0.1\right)$ with horizontal orientation & 0.8091 & 0.6903 & 0.8560 & - \\
\hline MC columns $\left(\varphi_{\text {mon }}=10.0\right)$ with horizontal orientation & 0.8283 & 0.6786 & 0.8211 & - \\
\hline MC aggregate-aggregate collection $\left(\varphi_{\text {mon }}=0.1\right)$ & 0.6988 & 0.5749 & 0.8280 & - \\
\hline Scaling relationship 1: $a^{\prime}=a+b+c, b^{\prime}=a+c, c^{\prime}=b+c$ & 0.6920 & 0.5550 & 0.8019 & 2.247 \\
\hline Scaling relationship 2: $a^{\prime}=a+b+(1 / 2) c, b^{\prime}=a+c, c^{\prime}=b+c$ & 0.7922 & 0.6951 & 0.8774 & 2.140 \\
\hline Scaling relationship 3: $a^{\prime}=a+b+c, b^{\prime}=a+c, c^{\prime}=a$ & 0.6823 & 0.4656 & 0.6823 & 2.148 \\
\hline Scaling relationship 4: $a^{\prime}=a+2 b+(1 / 4) c, b^{\prime}=a+c, c^{\prime}=a$ & 0.6126 & 0.4288 & 0.6999 & 2.332 \\
\hline
\end{tabular}

different scaling regimes as a way to parameterize the aggregation process.

\section{b. Ellipsoid fitting uncertainties}

One discrepancy in our comparison between simulated and observed aggregates has been the tendency for our simulated aspect ratio distributions to have mean values shifted more toward unity than the MASC derived ellipsoids. Some of this discrepancy can be explained due to uncertainties produced by the gradient descent algorithm used to estimate the best-fit ellipsoids. Jiang et al. (2019) tested their gradient descent algorithm on Monte Carlo-generated aggregates from the Ice Particle and Aggregate Simulator (IPAS) simulator (Schmitt and Heymsfield 2010; Przybylo et al. 2019). The results of this test are shown in Fig. 12 for both the histograms themselves (left column) and their ratio (right column). The overall bias introduced by the gradient descent estimate acts to reduce the apparent ellipsoid aspect ratios in a way that is more consistent with our Monte Carlo simulations. For example, $\varphi_{c b}$ derived from the gradient descent algorithm produces very few values near unity but the actual Monte Carlo-generated aggregates actually has more values closer to unity. The peak in the bivariate observations (Fig. 2) is therefore actually closer to the prolate line and shifted to higher values in a way that is more consistent with Fig. 5. This uncertainty, however, does not explain all differences between the observations and Monte Carlo-generated aggregates. Figure 12 suggests that the observed $\bar{\varphi}_{c a} \approx 0.4$ (Fig. 1) is shifted by about $20 \%$, that is, $\bar{\varphi}_{c a} \approx 0.48$. Many of our model runs show higher values, that is, $\bar{\varphi}_{c a} \approx 0.6$ (Figs. 7 and 8 ). However, this discrepancy can be explained from the existence of different scaling regimes as demonstrated in Table 1.

The main assumption for use of Eq. (7) in model calculations is that the aggregate $a$-axis lengths (or equivalently $V_{i_{0}}$ ) is statistically independent of $\varphi_{b a}$ and $\varphi_{c a}$. This allows for distribution moments of $V_{i}$ (or mass $\left.m_{i}\right)$ to simply be the product of each distributions' moments. However, if $a$ is correlated with $\varphi_{b a}$ and $\varphi_{c a}$, then the moments of $V_{i}$ could be significantly different. From the MASC database we found that the correlation coefficients between $a$ and each aspect ratio were very low. For instance, the correlation coefficient values for the NSA database are $\operatorname{corr}\left(a, \varphi_{b a}\right)=-0.0919$ and $\operatorname{corr}\left(a, \varphi_{c a}\right)=+0.0545$. These low values suggest that we can indeed consider the $a$-axis lengths and the aspect ratios to be independent of one another such that the volume (mass) distribution is accurate. The effects of estimating $V_{i}$ using Eq. (3) is shown in Fig. 13 where the $V_{i_{0}}$ and $V_{i}$ from the NSA MASC are shown along with two separate Monte Carlo estimates using Eq. (7). We first fit a Pareto distribution to the MASC dataset for $V_{i_{0}}$. This type of continuous distribution is similar to what a bulk microphysics model might assume. The second 


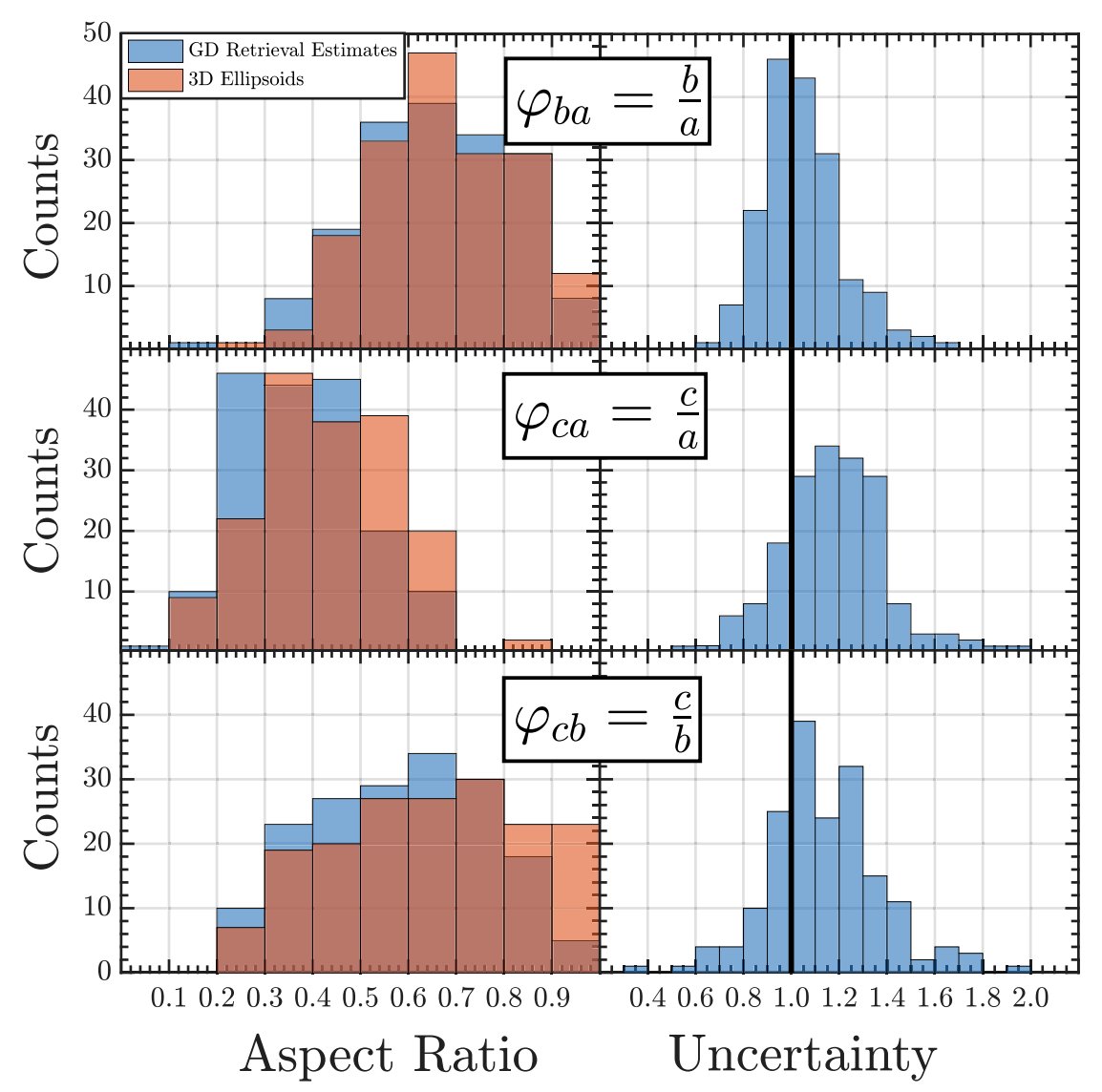

FIG. 12. Comparison of 3D ellipsoids estimated from Jiang et al. (2019) and the gradient descent (GD) retrieval estimates from the 2D ellipse projections from each ellipsoid. (left) Histograms of all counts $(N=176)$ for both ellipsoid estimates. (right) As in (left), but for their ratio (3D ellipsoid divided by the gradient descent retrieval estimates). Uncertainty values of 1.0 (thick black line) illustrate that both methods produce the same aspect ratio.

method uses a naive bootstrapping approach that randomly samples $V_{i_{0}}$ from the MASC NSA database. For each method, we then discretized Eq. (7) and sampled from the $V_{i_{0}}$ and aspect ratio distributions to estimate $V_{i}$. These estimated distributions are overall very similar to that derived from the MASC database despite the biases introduced by Monte Carlo and naive bootstrapping techniques.

\section{c. Fractal calculation uncertainties}

One concern with our spheroid aggregate results is whether our implicit aggregation modeling method yields different aggregates and therefore different fractal properties than the explicit methods of Westbrook et al. (2004b); Schmitt and Heymsfield (2010) and others. Differences between implicit and explicit aggregation might explain our very different prolate monomer results from that of Schmitt and Heymsfield (2010). Therefore, we test the robustness of our prolate results by comparing the evolution of mean fractal dimensions for monomer aspect ratios that overlap that of Schmitt and Heymsfield (2010). Both sets of results are shown in Fig. 14, where $\varphi_{\text {mon }}=2.0$ and $\varphi_{\text {mon }}=5.0$ are the aspect ratios used by Schmitt and Heymsfield (2010) for their hexagonal columns. For those monomer aspect ratios, our results compare well which suggests our methods are also comparable. To test whether our extended monomer aspect ratio ranges of $\varphi_{\text {mon }}=10$ and $\varphi_{\text {mon }}=20$ suffer from a lack of dipole resolution, we also reran the $\varphi_{\text {mon }}=10$ case by increasing the number of monomer dipoles by 3 times. However, we found that increasing the number of monomer dipoles did not significantly change the mean fractal dimension. Therefore, the high sensitivity of column aggregate $D_{f}$ to $\varphi_{\text {mon }}$ below that tested by Schmitt and Heymsfield (2010) seems to reflect the geometry of the aggregation problem rather than the way we compute $D_{f}$ or the assumptions we use. 


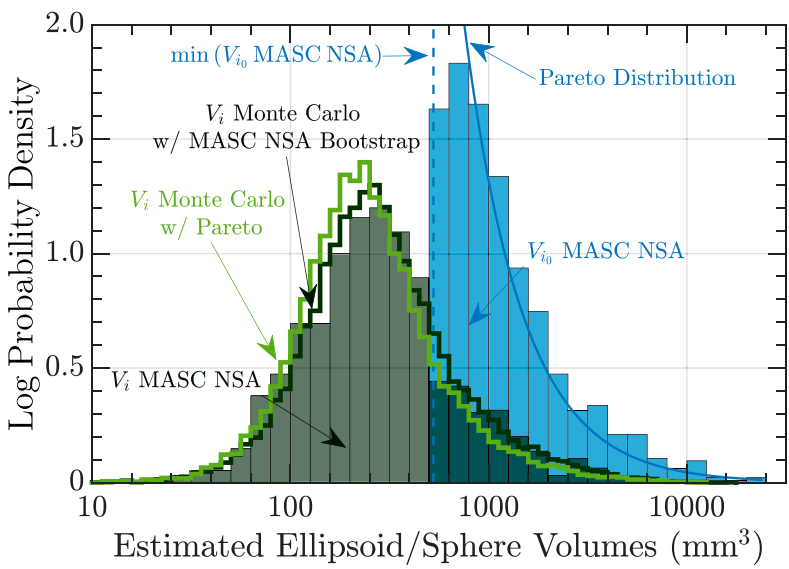

FIG. 13. Log probability density comparison of MASC derived sphere shells $V_{i_{0}}$ and ellipsoid shells $V_{i}$ with those estimated using the continuous bivariate beta distribution from Eq. (7). A Pareto distribution is used to estimate the probability density of $V_{i_{0}}$ from the MASC NSA dataset. We used 20000 samples from each distribution to generate the two $V_{i}$ estimates.

\section{Concluding remarks}

Westbrook et al. (2004b) conclude their Monte Carlo aggregation paper by stating: "In summary, we have a fairly complete understanding of the geometry of the atmospheric ice crystal aggregates... . The fact that the same evolution is seen for differing initial monomer populations (rods and rosettes) suggests that a single set of geometric relationships for ice aggregates can successfully be applied in a wide range of cloud conditions." Our results, however, suggest a less complete picture where the apparent geometric universality as simulated by Westbrook et al. (2004a,b) is a result of the small range of their tested monomer aspect ratios and their assumption that $D_{f}$ is sufficient for characterizing aggregate geometry. For instance, our results illustrate that fractal dimensions for thin column aggregates throughout evolution are much lower than the often reported $D_{f} \approx$ 2.0 , which even holds when $N_{\text {mon }} \geq 100$. For aggregates composed of very thin plates, $D_{f}$ increases from about $D_{f} \approx 2.0$ to $D_{f} \approx 2.1$ when $N_{\text {mon }}=100$. This suggests that the aggregate $D_{f}$ evolution takes much longer for very eccentric monomers (Fig. 11). The opposite seems to happen for lacunarity and density where the evolution is quicker for eccentric monomers but slower for more spherical monomers. For very eccentric particles $\left[\min \left(\varphi_{\mathrm{mon}}, \varphi_{\mathrm{mon}}^{-1}\right)=0.05\right]$ the majority of density changes seems to occur from $N_{\text {mon }}=2$ to $N_{\text {mon }}=3$.

Our results not only support those reported in previous studies (Fig. 14), but our tests also fall into a much larger range of fractal dimensions $\left(1.6 \leq D_{f} \leq 2.5\right)$ that happens to be consistent with that reported in Jiang and Logan (1991) for marine snow aggregated through differential sedimentation. ${ }^{2}$ While the aggregation physics between snow aggregates and marine snow aggregates are likely to be very different due to the difference in fall speed regimes, the techniques from these marine snow studies used to connect monomer properties to aggregate properties could be adapted for ice-ice aggregates as well. As shown in Table 1 from Logan and Kilps (1995), the relationship between the assumed monomer Euclidean geometry and the fractal properties of their aggregates seems to be directly related to a weighted average of monomer shape and packing factors. Similarly, the limiting behavior of fractal dimensions according to monomer aspect ratio mirrors the limiting behavior of spheroidal shape factors (see Fig. B1 from Harrington et al. 2013). As a result, it is likely possible to directly connect the fractal quantities of aggregates to the distribution of monomer properties. Aggregate properties therefore are likely to depend upon the prevalence of thin longer crystals such as needles or perhaps polycrystalline scrolls or twins.

The present work questions the universality of some mass-dimensional power-law framework assumptions. Particularly troublesome is the prefactor coefficient, $\alpha_{m}$, which is solved by assuming initial aggregates are uniformly filled spheres (Schmitt and Heymsfield 2010), by providing a best fit to the in situ data (Locatelli and Hobbs 1974; Brown and Francis 1995; Mitchell 1996), or by integrating the mass-dimensional relationship along with the in situ derived particle size distribution to match independent estimates of ice water content (Heymsfield and Westbrook 2010). Our numerical results show that these assumptions, while convenient for closing the system of equations in a microphysics model, are not necessarily consistent with the evolution of fractal and Euclidean geometric estimates. Initial aggregates show a wide variety of different fractal dimensions and lacunarities that evolve together as aggregates evolve. Although plate aggregates seem to consistently produce similar values of $D_{f}$, lacunarity is variable depending on different combinations of monomer sizes and shapes and generally increases from $N_{\text {mon }}=2$ to $N_{\text {mon }} \approx 25$. The lack of consistent behavior between $D_{f}$ and $\Lambda$ implies that a single $D_{f}$ value is not enough to completely characterize an aggregate's geometry. Interestingly enough, the mathematical approach for estimating $\alpha_{m}$ as used by Schmitt and Heymsfield (2010) led to better agreement of ice water content with observations than the empirical

\footnotetext{
${ }^{2}$ Marine snow is not to be confused with atmospheric ice-ice snow aggregates in marine environments. Rather, marine snow refers to biological material like fish excrement that drops from the surface of the ocean.
} 


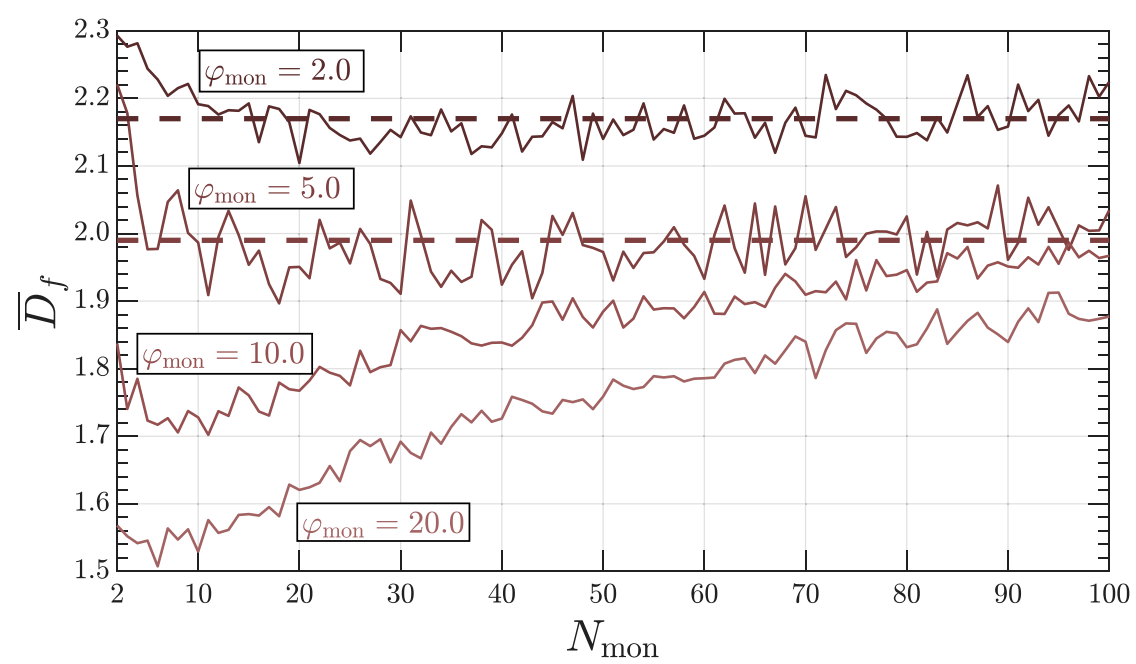

FIG. 14. Mean fractal dimension for prolate (column) aggregates. Dashed lines represent values reported by Schmitt and Heymsfield (2010) for monomer aspect ratios of 2.0 and 5.0.

methods of Heymsfield et al. (2004). Similarly, other mathematically based parameterization techniques could be used as well to utilize both measures of fractal dimension and lacunarity as described by Blumenfeld and Mandelbrot (1997) and Mandelbrot (1992). The use of lacunarity in model development is particularly attractive since lacunarity can be easily calculated without the need for any regression technique.

Microphysics models often parameterize particle properties in terms of an assumed maximum dimension. However, this simplification means that particles with the same maximum dimension will necessarily have the same masses and fall speeds. Passarelli (1978a,b), Passarelli and Srivastava (1979), Sasyo and Matsuo (1985), Böhm (1992), and others came up with clever ways to incorporate a dispersion of fall speeds for the same snow aggregate mass or dimension. The bivariate ellipsoidal aspect ratio model presented in this paper allows for a simple, analytical way to capture the appropriate fall speed and mass dispersion. Despite the correlation of shape and density with $N_{\text {mon }}$ (and therefore size), our estimates of the ellipsoid volume distribution are still consistent with that of observations (Fig. 13). This consistency suggests that aggregates at any particular size represent many different stages of aggregation. For distributions of sufficiently developed aggregates, the maximum dimension and shape can be considered separate and can be specified using different functions whereas the ellipsoid distribution can be represented using Eq. (7) as the average distribution of aggregate shapes. This would best work for plate aggregates since the mean aspect ratio quantities do not significantly change throughout most of the evolution (Figs. 7 and 8). It is already common for microphysics schemes to compute bulk quantities as ratios of gamma functions. The moment approach using Eqs. (8) and (10) yields a closed set of equations for evolving ellipsoid shape that fits neatly into the bulk paradigm, provided that $\bar{\varphi}_{b a}, \bar{\varphi}_{c a}$, and $\left\langle\varphi_{b a} \varphi_{c a}\right\rangle$ are parameterized during the aggregation process. Future aggregation work should focus on understanding the evolution and distributions of $N_{\text {mon }}$ and monomer aspect ratios since these factors can be used, in theory, to directly predict average aggregate morphology. Characterizing these monomer properties in models and observations will ensure appropriate estimations and parameterization of aggregate properties.

Finally, this paper reconciles historically inconsistent claims made about aggregate shapes. The early observations of Magono and Nakamura (1965) and later observations of Brandes et al. (2007) suggest snow aggregates are quasi-spherical whereas some radar results suggest aggregates are oblate (Moisseev et al. 2015) with aspect ratios of approximately 0.6 (Matrosov et al. 2005; Hogan et al. 2012; Garrett et al. 2015). In situ observations from Korolev and Isaac (2003) suggest aggregates have mean aspect ratios between 0.6 and 0.8 . It is therefore not surprising that these average $2 \mathrm{D}$ analogs are within the aspect ratio distributions of both observed (Figs. 1 and 2) and modeled (Figs. 5 and 9) aggregates. The bias toward aspect ratios of unity could be explained by projection uncertainties due to orientation. Therefore, the consistency of the 0.6 aspect ratio value derived from in situ observations and that used in homogeneous oblate spheroid radar approximations should be viewed as strictly coincidental since aggregates are rarely oblate.

Acknowledgments. Computations for this research were performed on the Pennsylvania State University's 
Institute for CyberScience Advanced CyberInfrastructure (ICS-ACI). This research was supported by the U.S. Department of Energy's Atmospheric Science Program Atmospheric System Research, an Office of Science, Office of Biological and Environmental Research program, under Grants DE-SC0018933 and DE-SC0013953.

\section{REFERENCES}

Bailey, M. P., and J. Hallett, 2009: A comprehensive habit diagram for atmospheric ice crystals: Confirmation from the laboratory, AIRS II, and other field studies. J. Atmos. Sci., 66, 28882899, https://doi.org/10.1175/2009JAS2883.1.

Blei, D. M., T. L. Griffiths, M. I. Jordan, and J. B. Tenenbaum, 2003: Hierarchical topic models and the nested Chinese restaurant process. Advances in Neural Information Processing Systems 16 (NIPS 2003), Whistler, BC, Canada, Neural Information Processing Systems Foundation, 17-24, https:// papers.nips.cc/paper/2466-hierarchical-topic-models-and-thenested-chinese-restaurant-process.

Blumenfeld, R., and B. B. Mandelbrot, 1997: Lévy dusts, MittagLeffler statistics, mass fractal lacunarity, and perceived dimension. Phys. Rev. E, 56, 112-118, https://doi.org/10.1103/ PHYSREVE.56.112.

Böhm, J., 1989: A general equation for the terminal fall speed of solid hydrometeors. J. Atmos. Sci., 46, 2419-2427, https://doi.org/ 10.1175/1520-0469(1989)046<2419:AGEFTT>2.0.CO;2.

_ 1992: A general hydrodynamic theory for mixed-phase microphysics. Part III: Riming and aggregation. Atmos. Res., 28, 103-123, https://doi.org/10.1016/0169-8095(92)90023-4.

Brandes, E., K. Ikeda, G. Zhang, M. Schönhuber, and R. M. Rasmussen, 2007: A statistical and physical description of hydrometeor distributions in Colorado snowstorms using a video disdrometer. J. Appl. Meteor. Climatol., 46, 634-650, https://doi.org/10.1175/JAM2489.1.

Brown, P. R. A., and P. N. Francis, 1995: Improved measurements of the ice water content in cirrus using a total-water probe. J. Atmos. Oceanic Technol., 12, 410-414, https://doi.org/10.1175/ 1520-0426(1995)012<0410:IMOTIW>2.0.CO;2.

Chen, J.-P., and D. Lamb, 1994: The theoretical basis for the parameterization of ice crystal habit: Growth by vapor deposition. J. Atmos. Sci., 51, 1206-1221, https://doi.org/10.1175/ 1520-0469(1994)051<1206:TTBFTP > 2.0.CO;2.

Chhabra, A., and R. V. Jensen, 1989: Direct determination of the $f(\alpha)$ singularity spectrum. Phys. Rev. Lett., 62, 1327-1330, https:// doi.org/10.1103/PhysRevLett.62.1327.

Connolly, P. J., C. Emersic, and P. R. Field, 2012: A laboratory investigation into the aggregation efficiency of small ice crystals. Atmos. Chem. Phys., 12, 2055-2076, https://doi.org/10.5194/acp12-2055-2012.

Dunnavan, E. L., and Z. Jiang, 2019: A general method for estimating bulk 2D projections of ice particle shape: Theory and applications. J. Atmos. Sci., 76, 305-332, https://doi.org/ 10.1175/JAS-D-18-0177.1.

Erfani, E., and D. L. Mitchell, 2016: Developing and bounding ice particle mass- and area-dimension expressions for use in atmospheric models and remote sensing. Atmos. Chem. Phys., 16, 4379-4400, https://doi.org/10.5194/acp-16-4379-2016.

Garrett, T. J., S. E. Yuter, C. Fallgatter, K. Shukurko, S. R. Rhodes, and J. L. Endries, 2015: Orientations and aspect ratios of falling snow. Geophys. Res. Lett., 42, 4617-4622, https://doi.org/ 10.1002/2015GL.
Gillespie, D. T., 1975: An exact method for numerically simulating the stochastic coalescence process in a cloud. J. Atmos. Sci., 32, 1977-1989, https://doi.org/10.1175/1520-0469(1975)032<1977: AEMFNS $>2.0 . \mathrm{CO} ; 2$.

Harrington, J. Y., K. Sulia, and H. Morrison, 2013: A method for adaptive habit prediction in bulk microphysical models. Part I: Theoretical development. J. Atmos. Sci., 70, 349-364, https:// doi.org/10.1175/JAS-D-12-040.1.

Hashino, T., and G. J. Tripoli, 2011a: The spectral ice habit prediction system (SHIPS). Part III: Description of the ice particle model and the habit-dependent aggregation model. J. Atmos. Sci., 68, 1125-1141, https://doi.org/10.1175/2011JAS3666.1.

_, and — 2011b: The spectral ice habit prediction system (SHIPS). Part IV: Box model simulations of the habit-dependent aggregation process. J. Atmos. Sci., 68, 1142-1161, https:// doi.org/10.1175/2011JAS3667.1.

Heymsfield, A. J., and C. D. Westbrook, 2010: Advances in the estimation of ice particle fall speeds using laboratory and field measurements. J. Atmos. Sci., 67, 2469-2482, https://doi.org/ 10.1175/2010JAS3379.1.

- A. Bansemer, C. Schmitt, C. Twohy, and M. R. Poellot, 2004: Effective ice particle densities derived from aircraft data. J. Atmos. Sci., 61, 982-1003, https://doi.org/10.1175/ 1520-0469(2004)061<0982:EIPDDF>2.0.CO;2.

Hogan, R. J., L. Tian, P. R. A. Brown, C. D. Westbrook, A. J. Heymsfield, and J. D. Eastment, 2012: Radar scattering from ice aggregates using the horizontally aligned oblate spheroid approximation. J. Appl. Meteor. Climatol., 51, 655-671, https:// doi.org/10.1175/JAMC-D-11-074.1.

Ishimoto, H., 2008: Radar backscattering computations for fractalshaped snowflake. J. Meteor. Soc. Japan, 86, 459-469, https:// doi.org/10.2151/jmsj.86.459.

Jensen, A. A., J. Y. Harrington, H. Morrison, and J. A. Milbrandt, 2017: Predicting ice shape evolution in a bulk microphysics model. J. Atmos. Sci., 74, 2081-2104, https://doi.org/10.1175/ JAS-D-16-0350.1.

Jiang, Q., and B. E. Logan, 1991: Fractal dimensions of aggregates determined from steady-state size distributions. Environ. Sci. Technol., 25, 2031-2038, https://doi.org/10.1021/es00024a007.

Jiang, Z., M. Oue, J. Verlinde, E. Clothiaux, K. Aydin, G. Botta, and Y. Lu, 2017: What can we conclude about the real aspect ratios of ice particle aggregates from two-dimensional images? J. Appl. Meteor. Climatol., 56, 725-734, https://doi.org/10.1175/ JAMC-D-16-0248.1.

-, J. Verlinde, E. E. Clothiaux, K. Aydin, and C. Schmitt, 2019: Shapes and fall orientations of ice particle aggregates. J. Atmos. Sci., 76, 1903-1916, https://doi.org/10.1175/JASD-18-0251.1.

Karperian, A., 2013: FracLac for ImageJ-FracLac Advanced User's Manual, version 2.5. https:/imagej.nih.gov/ij/plugins/ fraclac/FLHelp/Introduction.htm.

Kleinkort, C., G.-J. Huang, V. N. Bringi, and B. M. Notaros, 2017: Visual hull method for realistic 3D particle shape reconstruction based on high-resolution photographs of snowflakes in free fall from multiple views. J. Atmos. Oceanic Technol., 34, 679-702, https://doi.org/10.1175/JTECHD-16-0099.1.

Korolev, A., and G. Isaac, 2003: Roundness and aspect ratio of particles in ice clouds. J. Atmos. Sci., 60, 1795-1808, https://doi.org/ 10.1175/1520-0469(2003)060<1795:RAAROP > 2.0.CO; .

Locatelli, J. D., and P. V. Hobbs, 1974: Fall speeds and masses of solid precipitation particles. J. Geophys. Res., 79, 2185-2197, https://doi.org/10.1029/JC079i015p02185. 
Logan, B. E., and J. R. Kilps, 1995: Fractal dimensions of aggregates formed in different fluid mechanical environments. Water Res., 29, 443-453, https://doi.org/10.1016/0043-1354(94)00186-B.

Magono, C., and T. Nakamura, 1965: Aerodynamic studies of falling snowflakes. J. Meteor. Soc. Japan, 43, 139-147, https://doi.org/ 10.2151/jmsj1965.43.3_139.

Mandelbrot, B. B., 1992: Plane DLA is not self-similar; Is it a fractal that becomes increasingly compact as it grows? Physica A, 191, 95-107, https://doi.org/10.1016/0378-4371(92)90511-N.

- 1994: A fractal's lacunarity, and how it can be tuned and measured. Fractals in Biology and Medicine, T. F. Nonenmacher, G. A. Losa, and E. R. Weibel, Eds., Springer, 8-21.

Maruyama, K.-I., and Y. Fujiyoshi, 2005: Monte Carlo simulation of the formation of snowflakes. J. Atmos. Sci., 62, 1529-1544, https://doi.org/10.1175/JAS3416.1.

Matrosov, S. Y., A. J. Heymsfield, and Z. Wang, 2005: Dualfrequency radar ratio of nonspherical atmospheric hydrometeors. Geophys. Res. Lett., 32, L13816, https://doi.org/ 10.1029/2005GL023210.

Mitchell, D. L., 1988: Evolution of snow-size spectra in cyclonic storms. Part I: Snow growth by vapor deposition and aggregation. J. Atmos. Sci., 45, 3431-3451, https://doi.org/10.1175/ 1520-0469(1988)045<3431:EOSSSI $>2.0$.CO;2.

_ 1996: Use of mass- and area-dimensional power laws for determining precipitation particle terminal velocities. J. Atmos. Sci., 53, 1710-1723, https://doi.org/10.1175/1520-0469(1996) $053<1710$ :UOMAAD $>2.0 . \mathrm{CO} ; 2$.

Moisseev, D. N., S. Lautaportti, J. Tyynela, and S. Lim, 2015: Dualpolarization radar signatures in snowstorms: Role of snowflake aggregation. J. Geophys. Res. Atmos., 120, 12 644-12 655, https://doi.org/10.1002/2015JD023884.

Morrison, H., and J. A. Milbrandt, 2015: Parameterization of cloud microphysics based on the prediction of bulk ice particle properties. Part I: Scheme description and idealized tests. J. Atmos. Sci., 72, 287-311, https://doi.org/10.1175/JAS-D-14-0065.1.

Nadarajah, S., and S. Kotz, 2005: Some bivariate beta distributions. Statistics, 39, 457-466, https://doi.org/10.1080/02331880500286902.

Nelson, J., 2001: Growth mechanisms to explain the primary and secondary habits of snow crystals. Philos. Mag. A, 81, 23372373, https://doi.org/10.1080/01418610108217152.

Passarelli, R. E., 1978a: An approximate analytic model of the vapor deposition and aggregation growth of snowflakes. J. Atmos. Sci., 35, 118-124, https://doi.org/10.1175/1520-0469(1978)035<0118: AAAMOT $>2.0 . \mathrm{CO} ; 2$.

— 1978b: Theoretical and observational study of snow-size spectra and snowflake aggregation efficiencies. J. Atmos. Sci., 35, 882-889, https://doi.org/10.1175/1520-0469(1978)035<0882: TAOSOS $>2.0 . \mathrm{CO} ; 2$.

— gregation theory. J. Atmos. Sci., 36, 484-493, https://doi.org/ 10.1175/1520-0469(1979)036<0484:ANAOSA > 2.0.CO;2.

Petty, G. W., and W. Huang, 2010: Microwave backscatter and extinction by soft ice spheres and complex snow aggregates. J. Atmos. Sci., 67, 769-787, https://doi.org/10.1175/ 2009JAS3146.1.

Pruppacher, H. R., and J. D. Klett, 1997: Microphysics of Clouds and Precipitation. Kluwer Academic Publishers, 975 pp.
Przybylo, V. M., K. Sulia, C. G. Schmitt, and Z. J. Lebo, 2019: The Ice Particle and Aggregate Simulator (IPAS). Part I: Extracting dimensional properties of ice-ice aggregates for microphysical parameterization. J. Atmos. Sci., 76, 1661-1676, https://doi.org/ 10.1175/JAS-D-18-0187.1.

Sasyo, Y., and T. Matsuo, 1985: Effects of the variations of falling velocities of snowflakes on their aggregation. J. Meteor. Soc. Japan, 63, 249-261, https://doi.org/10.2151/jmsj1965.63.2_249.

Schmitt, C. G., and A. J. Heymsfield, 2010: The dimensional characteristics of ice crystal aggregates from fractal geometry. J. Atmos. Sci., 67, 1605-1616, https://doi.org/10.1175/2009JAS3187.1.

Smith, T. G., G. D. Lange, and W. B. Marks, 1996: Fractal methods and results in cellular morphology-Dimensions, lacunarity and multifractals. J. Neurosci. Methods, 69, 123-136, https:// doi.org/10.1016/S0165-0270(96)00080-5.

Stein, T. H. M., C. D. Westbrook, and J. C. Nicol, 2015: Fractal geometry of aggregate snowflakes revealed by triple-wavelength radar measurements. Geophys. Res. Lett., 42, 176-183, https:// doi.org/10.1002/2014GL062170.

Thompson, G., P. R. Field, R. M. Rassmussen, and W. D. Hall, 2008: Explicit forecasts of winter precipitation using an improved bulk microphysics scheme. Part II: Implementation of a new snow parameterization. Mon. Wea. Rev., 136, 50955115, https://doi.org/10.1175/2008MWR2387.1.

van Opheusden, J., 1998: The origin of an increasing or decreasing multifractal spectrum. Physica A, 252, 10-22, https://doi.org/ 10.1016/S0378-4371(97)00626-2.

_ fractal spectrum of aggregating Lennard-Jones particles with Brownian dynamics. Physica A, 227, 183-196, https://doi.org/ 10.1016/0378-4371(95)00413-0.

Vicsek, T., F. Family, and P. Meakin, 1990: Multifractal geometry of diffusion-limited aggregates. Europhys. Lett., 12, 217-222, https://doi.org/10.1209/0295-5075/12/3/005.

Warren, P. B., and R. C. Ball, 1989: Anisotropy and the approach to scaling in monodisperse reaction-limited cluster-cluster aggregation. J. Phys. Math. Gen., 22, 1405-1413, https:// doi.org/10.1088/0305-4470/22/9/027.

Westbrook, C. D., and E. K. Sephton, 2017: Using 3-D-printed analogues to investigate the fall speeds and orientations of complex ice particles. Geophys. Res. Lett., 44, 7994-8001, https:// doi.org/10.1002/2017GL074130.

— R. C. Ball, P. R. Field, and A. J. Heymsfield, 2004a: A theory of growth by differential sedimentation, with application to snowflake formation. Phys. Rev. E, 70, 021403, https://doi.org/ 10.1103/PHYSREVE.70.021403.

$\longrightarrow, \ldots, \ldots$, and $\_, 2004 \mathrm{~b}$ : Universality in snowflake aggregation. Geophys. Res. Lett., 31, L15104, https://doi.org/ 10.1029/2004GL020363.

,$- \ldots$, and,- 2006 : Radar scattering by aggregate snowflakes. Quart. J. Roy. Meteor. Soc., 132, 897-914, https://doi.org/ 10.1256/qj.05.82.

_, R. J. Hogan, and A. J. Illingworth, 2008: The capacitance of pristine ice crystals and aggregate snowflakes. J. Atmos. Sci., 65, 206-218, https://doi.org/10.1175/2007JAS2315.1.

Wolf, M., 1996: Multifractality of snowflakes. Fractals, 4, 477-493, https://doi.org/10.1142/S0218348X96000595. 